\title{
Secuencia de enseñanza-aprendizaje de las ciencias experimentales en 5 años a través del juego
}

\section{Teaching-learning sequence of experimental sciences in 5 years of age through games}

\author{
DOI: $10.46932 / \mathrm{sfjdv2n5-116}$
}

Received in: Oct 1st, 2021

Accepted in: Dec 30th, 2021

\author{
Cristina Lara Zarcero \\ Graduada En Educación Infantil \\ RG FORMACIÓN \\ C/ Gabriel García Márquez, N², Murcia \\ Correo electrónico. investigaacionrgformacion@gmail.com
}

\section{RESUMEN}

El objetivo principal de este Trabajo Fin de Grado es diseñar una secuencia de enseñanza-aprendizaje de las ciencias experimentales, dividida en cuatro fases, la cual se ha puesto en práctica y se ha evaluado. Esta se ha llevado a cabo en un aula de cinco años del segundo ciclo de la Educación Infantil, donde se ha pretendido trabajar la relación que existe entre un objeto y el tipo de fuerza aplicada, su comportamiento y su utilización en la vida cotidiana. Tratando de este modo iniciarlos en el método científico. En cuanto a la metodología que se ha empleado ha sido el juego, que tiene en cuenta el desarrollo integral del alumnado, donde se han planificado actividades que les lleven no solo a entretenerse sino a ir más allá experimentando de manera directa y vivenciada en la adquisición de los nuevos aprendizajes. Por último, se ha realizado una evaluación inicial, continúa y final usando diferentes instrumentos de evaluación (entrevistas, murales y rubricas), donde los resultados han resultado ser satisfactorios, por lo que se demuestran que es posible evolucionar desde modelos estructurales científicos sencillos hacia más complejos.

Palabras clave: Juego educativo, Educación Infantil, conocimiento del entorno, ciencias experimentales y secuencia de enseñanza-aprendizaje.

\begin{abstract}
The main objective of this Final Degree Project is to design a teaching-learning sequence of experimental sciences, divided into four phases, which has been put into practice and evaluated. This has been carried out in a classroom of five year olds in the second cycle of Childhood Education, where the aim has been to work on the relationship between an object and the type of force applied, its behavior and its use in daily life. Trying in this way to initiate them in the scientific method. As for the methodology used has been the game, which takes into account the integral development of students, where activities have been planned to take them not only to entertain but to go beyond experimenting in a direct and experienced way in the acquisition of new learning. Finally, an initial, continuous and final evaluation has been carried out using different evaluation instruments (interviews, murals and rubrics), where the results have been satisfactory, showing that it is possible to evolve from simple to more complex scientific structural models.
\end{abstract}

Keywords: Educational game, Childhood Education, knowledge of the environment, experimental sciences and teaching-learning sequence. 


\section{MARCO TEÓRICO}

Algunos docentes piensan que trabajar el tema de las ciencias en educación infantil es complejo (Cantó, de Pro y Solbes, 2016), ya que es una pérdida de tiempo iniciar el aprendizaje científico, desde una temprana edad. Otros maestros piensan que los niños son capaces de realizar prácticas científicas y aprender ciencias (Spektor-Levy, Kesner Baruch y Mevarech, 2013). Pero si se tiene en cuenta esta última opinión, se podrá concebir al niño como un ser competente, activo y con potencialidades. Además tal y como señala Montessori (citado en Morales, 2015):

(...) la educación entre los seis y los doce años no es una continuación de la etapa anterior, pero se basa en dicha etapa. Dice que "en los psicológico, cambia decididamente la personalidad, y conforme lo ha determinado la naturaleza, durante este periodo el niño adquiere todo cuanto tiene que ver con la cultura (...). (p.294)

Por otro lado, parte del alumnado llega a la escuela con conocimientos previos respecto al tema del Universo. Por ello, es necesario brindarles actividades que estén adaptadas y traten de despertar su interés, y así su foco de atención en relación con este tema no se perderá para siempre, sino que los conocimientos los asimilara de manera organizada y sistemática. Y para tratar de captar este interés no hay porque ofrecerle datos aislados, sino que es necesario mostrarle nociones de naturaleza, siempre adaptadas a sus capacidades cognitivas. (Montessori citado en Morales, 2015).

También los docentes piensan que el tema del Universo no se puede abordar por ser un tema poco cercano al niño, y porque no podrán desarrollar un aprendizaje directo. Por esta razón, muchas docentes al tener escasas evidencias de este campo, basan su metodología en libros de texto, pero aun así tal y como dice Lanciano citado en Varela, Pérez, Ulla y Arias (2012) el uso de los libros de texto no garantiza un buen aprendizaje por ser actividades que no tienen cierta conexión con la observación. Para dar respuesta a este problema se va a dar la oportunidad al aula de 5 años, de observar y experimentar de manera directa con una canica, simulando que es un cohete propulsado en la cual se va aplicar tres fuerzas: humana, aire y agua.

Asimismo, como el niño desde que nace hasta que va madurando está en contacto con la naturaleza. Esto lo ha llevado a lo largo de la etapa de su infancia a observar el mundo que le rodea y a hacerse infinidades de preguntas, como por ejemplo, que hay después del cielo o cómo ir a la Luna. Son cuestiones que surgen y que ellos mismos se dan soluciones ajustándolas a su mundo, que dista mucho de la realidad. Por eso mismo en este trabajo fin de grado, se va dar también respuestas a estas preguntas.

Estas respuestas que se dan los niños a sí mismos son los denominados modelos explicativos. Para que estas respuestas se conviertan en modelos explicativos verdaderamente científicos se va utilizar una metodología activa: el juego. Pues bien y como dice Hirsh-Pasek y Golinkoff (citado en Fernández y 
García, 2008), la forma más adecuada de desarrollar aprendizajes científicos en las primeras etapas, para posteriormente evitar conductas de frustración o miedo al fracaso, es introducir planteamientos lúdicos. Es más se puede comprobar como a través de un estudio realizado por Arcos y Vigil (citado en Melo y Hernández, 2014) donde se hacían talleres de demostraciones y experimentos en el museo de Costa Rica, los visitantes estaban más atentos y dispuestos a colaborar en las actividades lúdicas, alejadas de la propuesta curricular. A partir de esta experiencia, el museo se ha visitado más y estas actividades han tenido más sentido en relación con los contenidos educativos. Sin cambiar de juego ni actividades se puede confirmar que los visitantes aprendían divirtiéndose. Por ello, tal y como justifica Fröbel (citado en Sanchidrián et al., 2010) el juego se presentará no solo como un pasatiempo sino como algo más allá de la simple diversión. De esta manera, los niños favorecerán el aprendizaje global (cognitivo, social , afectivo y motor), donde buscarán dar respuestas a los problemas que se plantean, para comprender el entorno que les concierne, a la misma vez que se sentirán motivados a lo largo de todo el proceso. Por lo tanto, aquí se refleja mi objeto de estudio de este trabajo de fin de grado (TFG), que es diseñar una secuencia de enseñanza-aprendizaje de las ciencias experimentales de cohetes propulsados, empleando como metodología el juego.

Para enseñar esta secuencia de enseñanza-aprendizaje de ciencias experimentales, desde una perspectiva investigativa, se va a seguir la estructura del ciclo de aprendizaje de evaluación, regulación y autorregulación de Jorba y Sanmartí citado en Gallego, Quinceno y Pulgarín (2014):

-Fase de exploración o de explicitación inicial: sitúa al alumno en el objeto de estudio y busca captar su atención; a la vez que permite detectar sus conocimientos previos. En esta fase se desarrollan actividades que ayuden a los estudiantes a formular preguntas previas e hipótesis desde situaciones, vivencias e intereses cercanos.

-Fase de introducción de los nuevos conocimientos: centrada en observar, comparar o relacionar cada situación que el estudiante ha captado previamente, de manera que se anime a interactuar con el material de estudio, con sus iguales y con el docente, buscando elaborar conceptos más significativos.

- Fase de estructuración y síntesis de los nuevos conocimientos: trata de ayudar al estudiante a construir su conocimiento como consecuencia de la interacción con el maestro y los compañeros.

-Fase de aplicación: permiten al estudiante aplicar los conocimientos adquiridos en otras situaciones similares de la vida cotidiana. (Jorba y Sanmartí citado en Gallego, Quinceno y Pulgarín, 2014, p. 926-927)

Dentro de la fase de introducción de nuevos conocimientos, cabe mencionar que se encuentran presentes:

"las actividades de investigación donde los alumnos trabajan siguiendo estrategias de trabajo similar a la que utilizan los científicos en la resolución de problemas. Los pasos de una investigación en Educación infantil son: 
1. Planteamiento de un problema.

2. Propuesta de soluciones

3. Diseño de un experimento.

4. Realización del experimento y recogida de resultados.

5. Conclusiones de la investigación" (Baños, 2017, p.6).

De esta manera, en este proyecto se va hacer un experimento usando la metodología del juego para trabajar el movimiento de una canica, imaginando que es un cohete propulsado. Para que este objeto realice una trayectoria vamos a apoyarnos en la primera ley de Newton o la denominada ley de Inercia, donde explica que para que un cuerpo salga de su estado de reposo, es necesario que una fuerza actué sobre él. (Vila y Sierra, 2008). Así pues, se van aplicar tres fuerzas: la canica se moverá si soplamos, la canica se moverá si usamos el cuerpo y la canica se moverá si inyectamos agua. Esta secuencia va tener una estrecha conexión con la secuencia de Mazas, Gil, Martínez, Hervás y Muñoz (2018), la cual parte de dos experimentos para explicar el comportamiento del agua y del aire.

En resumen, es importante estudiar el tema del Universo en Educación infantil aprendiendo desde una perspectiva activa, ya que a través de este aprendizaje los alumnos podrán manipular, experimentar y ensayar con materiales de estudio, obteniendo nueva información de su entorno y estableciendo relaciones con los mismos.

En cuanto a su presencia curricular se encuentra en todas las áreas del Decreto 254/2008, de 1 de agosto, por el que se establece el currículo del Segundo Ciclo de la Educación Infantil en la Comunidad Autónoma de la Región de Murcia. Pero haciendo mayor hincapié en el ÁREA 2. CONOCIMIENTO DEL ENTORNO”, concretamente en el BLOQUE 1. Medio físico: elementos, relaciones y medida.

Por eso, los objetivos que se pretenden conseguir a través de la puesta en práctica y reflexionado sobre la secuencia de enseñanza-aprendizaje de título Nos movemos por el espacio son:

1. Conocer que para que un objeto deje de estar en situación de reposo, se debe aplicar una fuerza. En este caso para que una canica se mueva, simulando que es un cohete propulsado, tienen que actuar tres fuerzas: humana, aire y agua.

2. Descubrir que personajes históricos han conseguido viajar al Universo y llegar a la luna.

3. Concienciarse de que el problema de la contaminación está presente en el Universo, y esto puede provocar la destrucción de nuestro planeta sino ponemos una solución al respecto. 


\section{DESARROLLO}

\subsection{CONTEXTO Y PARTICIPANTES}

La secuencia de enseñanza-aprendizaje Nos movemos por el espacio se va a poner en práctica en un colegio de titularidad pública, situado en los límites del barrio de la Flota con el de Santiago y Zaraiche. Esta va a estar dirigida para alumnos del segundo ciclo de la educación infantil, especialmente para un aula de 5 años. El barrio donde está ubicado cuenta con un gran número de habitantes y se estima que seguirá aumentando a lo largo de los próximos años, ya que se trata de un barrio de expansión. Debido a esto, todos los que se incorporan a la institución educativa son de nacionalidad española, aunque últimamente han habido alumnos de nacionalidad extranjera y ucraniana.

El nivel socioeconómico de las familias es de clase media, dado que sus profesiones están destinadas al sector de servicios, y su formación académica es de grado medio y superior.

En cuanto a la estructura del centro, está dividido en tres pabellones. El primer pabellón es de Educación Infantil, por eso, dispone de un aseo compartido dentro de la misma aula y se encuentra en planta baja para facilitar el acceso a los más pequeños a un patio que está adaptado a sus edades. El segundo y el tercer pabellón son de Educación Primaria, y tiene un patio específico para este nivel donde los alumnos pueden jugar a diferentes juegos, además de un gimnasio polideportivo. Sin embargo, lo que tienen en común todas las aulas es que están equipadas de un proyector audiovisual, un ordenador y una pizarra digital, introduciendo de esta manera desde las edades más tempranas las TIC (tecnologías de la información y la comunicación).

El aula donde se va a desarrollar la secuencia de enseñanza-aprendizaje tiene esta estructura (véase Figura 1)

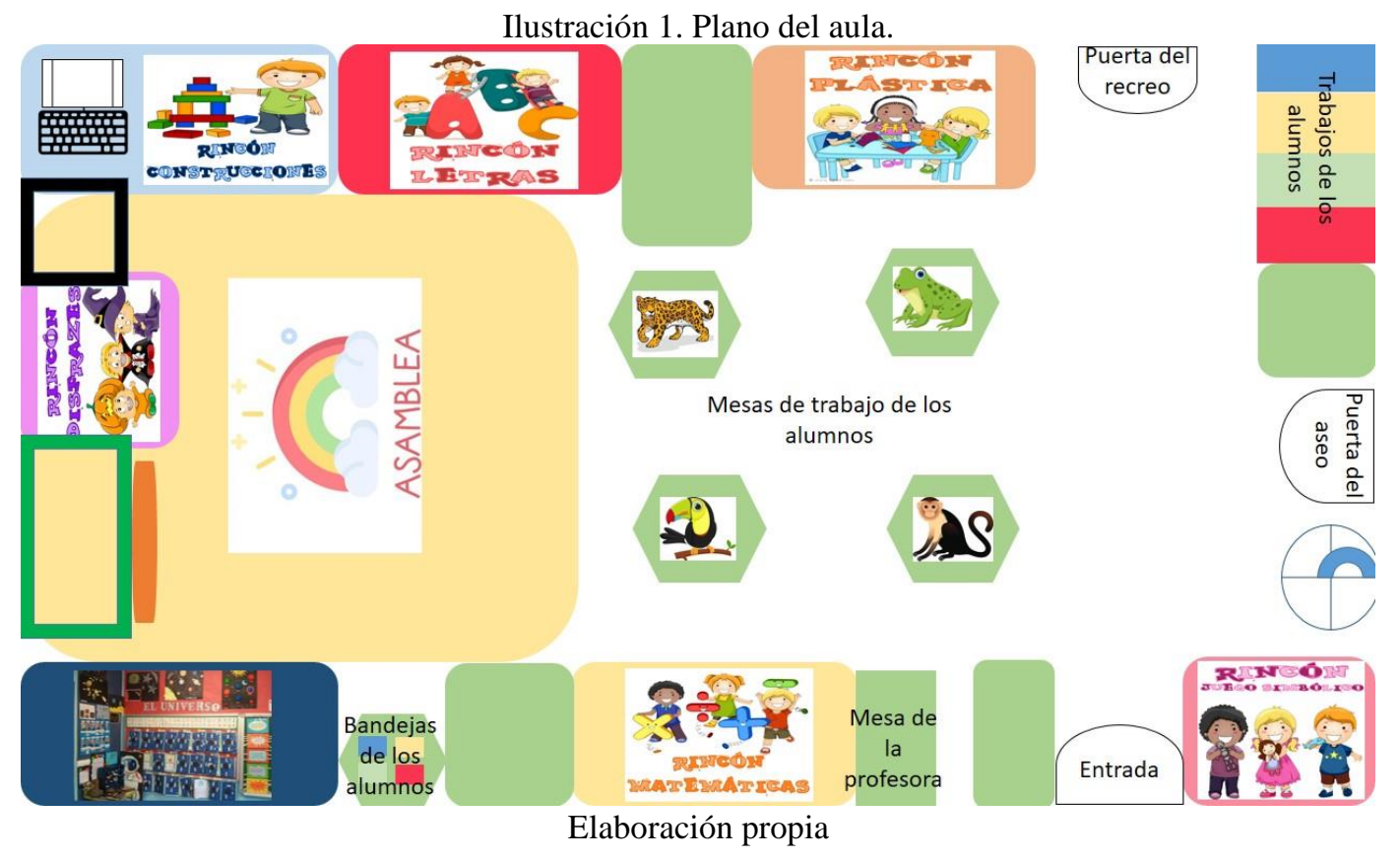


Para empezar el aula se compone de tres accesos: una puerta de entrada, lugar por donde los alumnos acceden todas las mañanas para comenzar con la jornada-tipo, y salen cuando finaliza esta. Una puerta del baño que se comunica con el aula de 5 años B. Y una puerta del recreo que da al patio, y en la cual se dirigen para descansar y jugar con los demás compañeros. El patio es un espacio abierto donde se va llevar a la práctica algunas actividades que va a permitir trasladar a los alumnos a otro contexto diferente, del que ya están acostumbrados.

También el aula está dividida en varias zonas, rincones, mobiliario y recursos. Aunque se va a prestar especial atención a aquellos que se van a utilizar para el desarrollo de la secuencia de enseñanza -aprendizaje.

Entre las distintas zonas que se puede encontrar, está la más conocida como Asamblea. Este lugar está destinado para realizar las rutinas diarias: pasar lista, contar el número de alumnos que han venido a clase, cantar la canción de los días de la semana y los meses del año, etc... Además se llevan a cabo todo tipo de actividades orales, cuentos y audiciones, y sobre todo se usa para la presentación y organización del trabajo que se va hacer.

Asimismo el aula se estructura en diferentes rincones: el rincón de los disfraces, el rincón de las construcciones, el rincón del lenguaje, el rincón de la plástica, el rincón de la lógica-matemática, el rincón de la casita y el rincón El Universo. En este rincón todos los materiales fabricados y que se utilizan en las actividades de la secuencia de enseñanza-aprendizaje, se destinan allí. La ventaja es que como se tratan de contenidos desconocidos para los alumnos les va a despertar curiosidad, ganas de manipularlos y descubrir sus características. Por lo tanto, cada vez que les toque jugar en este rincón se dirigirán al mismo y favorecerán la observación y la experimentación. Estos recursos permanecerán a lo largo de todo el tiempo hasta que finalice el proyecto del centro El espacio, que fue la base por el cual se diseñó ese rincón.

En cuanto al mobiliario, las mesas de trabajo se utilizan para que los alumnos almuercen, hablen con otros niños y sobre todo, para que hagan las tareas. En muchas ocasiones, se llama a los equipos de mesa (jaguar, tucanes, ranas y mono-tití) para realizar las actividades que suelen ser tanto individuales, en pequeños equipos y en gran grupo.

De acuerdo con los recursos materiales que dispone el aula, existen una gran variedad de materiales que se pudieron reutilizar y aprovechar, pero siempre y cuando diera su consentimiento la maestra-tutora. Por ello, se pudo planificar actividades atractivas para el grupo al que iba destinado. Para la actividad 2 se utilizó la luna, la tierra y el cohete espacial, que eran materiales que los alumnos habían elaborado y habían dejado en el rincón El Universo. Además en la actividad 3 y actividad 4 se usaron las bandejas para hacer una carrera, en la que pudieron practicar el movimiento de las canicas soplando con el aire e inyectando agua, además se realizó otra carrera, donde recogieron basura espacial. Finalmente, en la actividad 6 se necesitó la pizarra digital. El resto de materiales tales como lápices, ceras, rotuladores, temperas, tijera y pegamento fueron necesarios para poder abordar el resto de las actividades. 
En relación al nivel de vida que tienen los alumnos, es bastante bueno, dándose condiciones suficientes para que no haya problemas en el proceso de enseñanza-aprendizaje.

El grupo-clase de 5 años A, por el contrario, es bastante dinámico, ya que el aula lo forman 13 niñas y 11 niños siendo un total de 24 alumnos. Es decir, existe un alto nivel de diversidad sin contar con la presencia de alumnos con necesidades educativas especiales. Pero sí que existen alumnos con distintos ritmos de aprendizaje, desde los que tienen altos niveles de aprendizaje hasta los de dificultades de aprendizaje, debido a faltas de atención o lentitud en las tareas.

Por último, en la cohesión del grupo-clase se puede observar que a veces surgen conflictos entre compañeros. También hay cooperación, por ejemplo, cuando un alumno no entiende una actividad el otro compañero le ayuda. Aislamientos no existen, puesto que no se ha dado ningún caso de este comportamiento.

\subsection{PLANIFICACIÓN Y DISEÑO DE LA ACCIÓN DOCENTE}

La secuencia de enseñanza-aprendizaje Nos movemos por el espacio es una propuesta motivadora e interesante que se ha querido realizar en las Prácticas Escolares IV, las tres primeras semanas antes de dar las vacaciones de Semana Santa y Semana de primavera. En ella se han abordado todas las áreas del Decreto 254/2008, de 1 de agosto, por el que se establece el currículo del Segundo Ciclo de la Educación Infantil en la Comunidad Autónoma de la Región de Murcia, de manera interdisciplinar llevando a cabo la mayor parte de la implicación en el ÁREA 2. CONOCIMIENTO DEL ENTORNO”, concretamente en el BLOQUE 1. Medio físico: elementos, relaciones y medida. Así pues, los objetivos y contenidos curriculares que se han seleccionado de este documento curricular tienen una estrecha conexión con los objetivos y contenidos específicos que se pretenden trabajar en cada una de las actividades planificadas y quedan recogidos de la siguiente forma (véase Anexo I).

Por lo tanto, durante su puesta en práctica se va a fomentar el aprendizaje significativo creado por Ausubel, ya que se tratará de detectar las ideas previas y motivar a los alumnos en el tema del Universo para después introducir nuevos contenidos. De manera que relacionen los nuevos conocimientos con los que ya saben. También, reflexionarán sobre lo aprendido y lo aplicarán a otros contextos de la vida real, para establecer así una mejor calidad en dichas relaciones. Igualmente el Universo es un tema tan abstracto y difícil de entender para niños de estas edades, porque es un tema que no está a su alcance. Pero a la misma vez, es tan importante e interesante saber los problemas medioambientales que surgen en el planeta, aprender un poco de cultura y hechos sobre el mundo que les rodea. Para eso y para dar respuesta a las preguntas de los alumnos, se van a planificar distintas actividades que les permitan interactuar constantemente, iniciándolos de este modo en los contenidos de aprendizaje desde una metodología activa. 
Por lo tanto para llevar a la práctica la secuencia de enseñanza, se va a tener en cuenta las siguientes fases establecidas por Jorba y Sanmartí citado en Gallego, Quinceno y Pulgarín (2014), como hemos mencionado en líneas anteriores:

“-Fase de exploración o de explicitación inicial.

-Fase de introducción de los nuevos conocimientos.

-Fase de estructuración y síntesis de los nuevos conocimientos.

-Fase de aplicación” (Jorba y Sanmartí citado en Gallego, Quinceno y Pulgarín, 2014, p. 926-927).

\section{$\underline{\text { Temporalización }}$}

El tiempo necesario que se va a dedicar para poner en marcha la secuencia de enseñanzaaprendizaje va a ser desde el 18 hasta el 22 del de Marzo, coincidiendo así con uno de los proyectos del centro escolar titulado El espacio, que se llevará a cabo durante el segundo trimestre.

A continuación, se establece el horario del aula 5 años A, seleccionando aquellas franjas horarias en las que se va a desarrollar la secuencia de enseñanza-aprendizaje Nos movemos por el espacio (véase Figura 2)

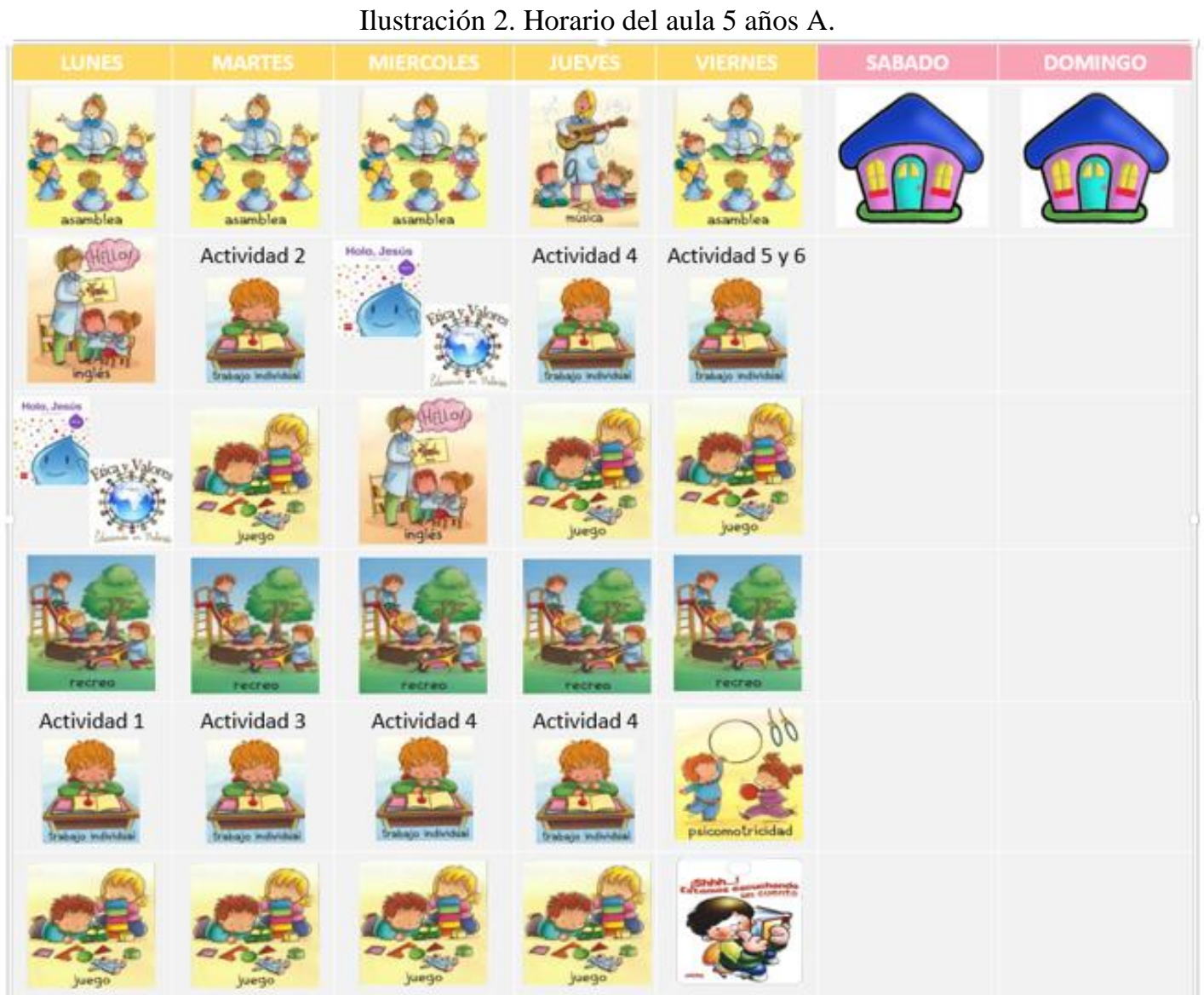

Elaboración propia. 
Desarrollo de las actividades

\subsubsection{Fase de exploración o de explicitación inicial}

2.2.1.1 Actividad 1. Nos convertimos en verdaderos astronautas de la NASA.

Los objetivos específicos que se pretenden desarrollar en esta actividad son:

- Intervenir activamente en el patio aportando ideas previas acerca del espacio.

- Participar en la creación del carnet de astronauta y tarjeta de la NASA.

Los contenidos específicos son:

- Conocimientos previos sobre el espacio. Formulación de hipótesis y expresión de opiniones e ideas.

- Expresión de la originalidad y la creatividad en el proceso de creación del carnet de astronauta y tarjeta de la NASA.

Después de las rutinas diarias, la maestra propondrá a los alumnos salir al patio y tumbarse en el suelo. Posteriormente, se les animará para que miren el cielo mientras se hacen preguntas que servirán para conocer las ideas previas (véase Anexo II).

Una vez detectados las ideas previas, volverán a la clase y se les motivará diciendo que nos vamos a convertir en verdaderos astronautas de la NASA. Para que esto se haga realidad, se explicará que es necesario crear antes nuestro carnet de astronautas para poder identificarnos, y tarjetas de la NASA para demostrar que formamos parte de esa estación espacial.

La actividad en principio se va hacer en gran grupo, en está los niños saldrán al patio. Luego se va realizar de forma individual, en la mesa de cada equipo donde elaboraran nuevos recursos.

Los materiales que van a ser necesarios son: el logotipo de la NASA (véase Anexo IV), tijeras, cartulinas, pinzas de colores (véase Anexo IV), ficha del carnet de astronauta (véase Anexo IV), fotografías de cada alumno, lápices, rotuladores, etc...

La duración será de aproximadamente de unos treinta minutos en total.

\subsubsection{Fase de introducción de los nuevos conocimientos}

2.2.2.1. Actividad 2. Conocemos historias espaciales y viajamos a la Luna.

Los objetivos específicos que se quieren conseguir en esta actividad son:

- Conocer y explicar algunos hechos y personas destacadas en la historia de la conquista espacial (Neil Armstrong, Edwin Aldrin y Michael Collins) a través del cuento: Apolo XI: Un viaje a la luna

- Colaborar en la dramatización del hito histórico: el primer hombre que piso la Luna.

Los contenidos específicos son:

- Aprendizaje y explicación de algunos hechos y personas destacadas en la historia de la conquista 
espacial (Neil Armstrong, Edwin Aldrin y Michael Collins) a través del cuento: Apolo XI: Un viaje a la luna

- Participación en la dramatización del hito histórico: el primer hombre que piso la Luna

La maestra les dirá a los alumnos, que se sienten en la Asamblea para escuchar un cuento sobre el primer hombre que piso la luna. Este cuento se titula Apolo XI: Un viaje a la luna (véase Anexo VII).

Después de la narración, la maestra les hará una serie de preguntas (véase Anexo VII) para saber si han entendido la historia, y si han aprendido algún conocimiento nuevo. Para reforzar los nuevos conocimientos y lograr que estos sean activos para el alumnado de educación infantil, saldrán al patio y realizarán una dramatización (véase Anexo VII) donde la maestra desempeñara el papel del primer astronauta que pisa la luna. Para mantener la atención de la clase, se les hará participes a los niños durante toda las escenas, de modo que ellos también viajarán junto con Neil Armstrong. Como durante su viaje encontrarán una piedra lunar, se las guardaran para después escribir sus iniciales con rotulador negro, y además se les dará la oportunidad de llevársela a sus casas y enseñársela a sus familiares.

Los alumnos, primero, se pondrán en semicírculo en la Asamblea y la profesora se colocará en el centro del banco para que todos escuchen con atención el cuento. En segundo lugar, formarán una fila en parejas simulando que están dentro del cohete espacial y se dirigirán al patio. Finalmente, de forma individual en sus mesas de equipo dibujaran sus iniciales en la piedra lunar.

Los materiales que se van a necesitar son el cuento de Apolo XI: Un viaje a la luna, el casco de astronauta (véase Anexo VII), la mochila de astronauta (véase Anexo VII), brazalete y cinturón plateado (véase Anexo VII), la luna, la Tierra, la bandera de Estados Unidos (véase Anexo VII), las tarjetas del logotipo de la NASA, los carnets de astronautas, piedras lunares, rotuladores negros, etc...

El tiempo necesario para esta actividad será de unos cuarenta minutos más o menos.

2.2.2.2 Actividad 3. Investigamos ¿Cómo puede moverse un cohete?

El único objetivo específico que se aborda en esta actividad es:

- Investigar y recoger datos sobre la fuerza humana, del aire y del agua: cohetes propulsados.

El contenido específico es:

- Indagación y recogida de datos sobre la fuerza humana, del aire y del agua: cohetes propulsados

Se va a realizar una investigación con los niños sobre el movimiento de los cohetes espaciales. Para ello nos guiaremos en los siguientes pasos.

\section{a. Problema a investigar}

El problema concreto que guiara la investigación sobre el movimiento será ¿un cohete puede $\underline{\text { moverse con la fuerza humana, del aire o del agua? }}$ 
Este problema se va resolver planteando un experimento donde se va a utilizar un cohete, que será una canica en el que se van aplicar distintas fuerzas (humana, aire y agua).

\section{b. Propuesta de soluciones}

Las propuestas de soluciones que pueden ofrecer los alumnos son las siguientes:

1. Un cohete puede moverse con fuego.

2. Un cohete puede moverse con la fuerza del viento.

3. Un cohete puede moverse si soplamos.

\section{c. Diseño experimental}

Este experimento para poder llevarlo a cabo se va a necesitar de un cohete, que será una canica y diferentes soportes (campos de fútbol, una pista de carreras y bandejas) para comprobar si el cohete puede moverse aplicando fuerzas (humana, aire y agua). Asimismo, el soporte aquí sí que varía pero el material no.

Durante la actividad, la docente dará una tabla que tendrán que rellenar una vez acabado el experimento.

Posteriormente, juntará a todos los alumnos en la Asamblea para comenzar con la experimentación. Para que esta sea divertida y entretenida para el aula se va hacer en forma de juego, de manera que todos se meterán de lleno en la investigación y querrán resolver la cuestión que se les plantea.

Para comenzar, se jugarán tres partidos de futbol donde irán pasando uno por uno, para vencer al jugador opuesto tendrán que hacer un clic en la canica con el dedo y meterla en la portería. Seguidamente, se hará una competición de carreras que irán participando de dos en dos, para ganar deberán de soplar fuerte la canica desde el punto de salida hasta la meta. Y por último, se pondrán cuatro bandejas en el suelo y se practicará a echar agua con una jeringuilla en la canica. En estos juegos se tratará de desarrollar la fuerza humana, del aire y del agua respectivamente.

\section{d. Realización de la investigación y recogida de datos}

Durante la realización de la experimentación se recogerán los datos en una tabla (ver Anexo IX). Una vez realizada la investigación y recogida los datos, éstos deberán ser interpretados para obtener los resultados. ¿Qué significa lo que hemos observado?

Los alumnos podrán observar que un cohete es capaz de moverse haciendo clic con el dedo en la canica (fuerza humana), soplando fuerte en la canica (fuerza del aire) e inyectando agua en la canica (fuerza del agua).

Los niños rellenarían la tabla anterior con los datos que han obtenido del experimento.

\section{e. Obtención de conclusiones}

Una vez hecho el experimento y registrado en la tabla los datos, la profesora se reunirá con el grupo-clase en la Asamblea y les hará una serie de preguntas como por ejemplo: ¿Un cohete puede 
moverse aplicando las tres fuerzas? ¿Y con la fuerza del agua también? ¿Con cuál creéis que ha resultado más complicado mover el cohete? ¿Y más fácil?

En el experimento, los niños se colocarán en la Asamblea en gran grupo, situándose de espaldas a la pizarra, y la docente se quedará en el banco. Luego, se sentarán en sus equipos de mesa e individualmente rellenarán las observaciones en la tabla.

Los materiales que se necesitan para llevar a cabo la investigación son: canicas, campos de fútbol hechos a cartulina, cuerdas, jeringuillas, bandejas de varios colores (rojo, verde, azul y amarillo), ficha del registro de la investigación sobre las tres fuerzas (véase Anexo X), etc...

El experimento se va a durar una hora como mucho.

2.2.2.3 Actividad 4. Descubrimos el gran problema de la basura espacial.

Los objetivos específicos que se quieren desarrollar en esta actividad son:

- Entender y ordenar la película de Wall-E: el batallón de limpieza.

- Colaborar en la construcción del transporte espacial recoge basura.

- Utilizar la fuerza del aire para mover un transporte espacial recoge basura y resolver el problema de la basura espacial mediante el juego Una guerra de limpieza.

Los contenidos específicos son:

- Comprensión y secuenciación de las escenas de la película de Wall-E: El batallón de limpieza.

- Implicación en la construcción del transporte espacial recoge basura.

- Utilización de la fuerza del aire para mover un transporte espacial recoge basura y resolución del problema de la basura espacial mediante el juego Una guerra de limpieza.

Los alumnos se sentaran en la Asamblea, y la profesora pondrá en la pizarra digital la película de Wally - E: El batallón de limpieza. El grupo deberá prestar mucha atención a la reproducción del vídeo, para después saber hacer la otra tarea. Seguidamente, se pondrán unos flashcards sobre la película (véase Anexo XIII) pegados en la pizarra y se tendrán que ordenar según la consigna "va después de".

Tras concienciarse del problema existente en la actualidad, los alumnos se dirigirán a sus equipos de mesa. A continuación, se les dará un dibujo de un transporte espacial recoge basura en formato de cartulina (véase Anexo XIII) para que lo decoren a su gusto y lo recorten por la línea de puntos. Después, se acercarán a la mesa de la maestra, y esta les pegará detrás una pajita fina y colocará encima otra más gruesa. Una vez terminado, podrán practicar en la Asamblea el movimiento que hace el transporte aplicando la fuerza del aire.

Posteriormente, se explicará que se va hacer un juego que se llama Una guerra de limpieza donde nos imaginamos que somos astronautas y vamos montados en el transporte recoge basura. Ahora, para 
moverlo necesitamos usar la fuerza de aire, vamos viajando por el espacio, y justo caemos en un punto concreto donde hay basura, tenemos que recogerla. De nuevo volvemos a movernos y paramos en otra zona donde hay basura, la cogemos y así sucesivamente. El objetivo del juego es recolectar la mayor cantidad de basura espacial en poco tiempo, y gana el primero que consiga llenar su bandeja.

En primer lugar, los alumnos estarán organizados en semicírculo y la maestra estará sentada a la izquierda en una silla, luego esta se pondrá enfrente. Después, los alumnos se dirigirán a sus equipos de trabajo y realizaran de forma individual transportes recoge basura. Cuando hayan acabado, se hará un juego en gran grupo en la Asamblea donde irán saliendo, de cuatro en cuatro, para competir.

Los materiales que se necesitan para poner en marcha esta actividad son: la película de Wally-E: El batallón de limpieza, flashcards, dibujo de un transporte espacial recoge basura en formato cartulina, ceras manley, tijeras, pajitas de diferentes grosores (fina y gruesa), materiales no utilizables y bandejas de varios colores (rojo, verde, azul y amarillo).

El tiempo para ponerla en práctica es de tres días, dedicando una hora cada día.

\subsubsection{Fase de estructuración y síntesis de los nuevos conocimientos}

\subsubsection{Actividad 5. Sabemos más de lo que creemos sobre el espacio}

El objetivo específico que se quiere alcanzar es:

- Reflexionar colectivamente sobre los conocimientos adquiridos respecto al espacio y obtener conclusiones acerca de lo aprendido en un mural.

El contenido específico es:

- Razonamiento en colectivo sobre los conocimientos adquiridos respecto al espacio y obtiene conclusiones acerca de lo aprendido en un mural.

Desde el principio de la secuencia de enseñanza-aprendizaje, se pegará en la pizarra una cartulina de color azul que los alumnos irán rellenando conforme vayan aprendiendo nuevos conocimientos (véase Anexo VX). Una vez que hayan completado el mural, la maestra ira llamando por equipos para que se sienten en la Asamblea, luego se recordará entre todos los contenidos que se han ido abordando. Ahora, se hará un debate con el grupo-clase para sacar las conclusiones, que la docente recogerá en otra cartulina de color amarilla (véase Anexo VX).

Durante toda la actividad los niños estarán en gran grupo, distribuidos en semicírculo en la Asamblea y la profesora colocada en el banco frente a ellos.

Para llevar a cabo esta actividad se va a necesitar los siguientes materiales: el mural con imagenes de los contenidos aprendidos y una cartulina grande de color amarillo.

La duración de esta actividad será de unos veinte minutos. 


\subsubsection{Fase de aplicación}

2.2.4.1. Actividad 6. Aplicamos lo que hemos aprendido en el Kahoot

Los objetivos específicos que se quieren conseguir en esta actividad son:

- Aplicar lo aprendido a otras situaciones de la vida cotidiana mediante el juego Kahoot.

Los contenidos específicos son:

- Aplicación de lo aprendido a otras situaciones de la vida cotidiana mediante el juego Kahoot.

Se pondrá en la pizarra digital un juego que se llama Kahoot sobre el tema del Universo (véase Anexo VXI), que consiste en un cuestionario con cuatro posibles respuestas representadas por figuras geométricas (rectángulo, cuadrado, triangulo y círculo). Para que toda la clase participe, se dará a cada alumno cuatro tarjetas con estas formas geométricas y los niños irán levantando conforme consideren cual es la respuesta correcta. De esta manera, aplicaran lo aprendido a otros contextos diferentes de la vida.

En cuanto al agrupamiento de la actividad, los niños se distribuirán en gran grupo, situados en semicírculo en la Asamblea y la profesora sentada en el banco enfrente de ellos.

De materiales solo se va a necesitar la pizarra digital, el juego Kahoot y las tarjetas con formas geométricas.

En cuanto a la duración de la actividad, se estima que durará unos 20 minutos.

\subsection{EVALUACIÓN}

Antes de pasar a comentar los tipos de instrumentos de evaluación que se van a usar para evaluar esta secuencia de enseñanza-aprendizaje, se hace necesario aclarar, bien como especifica el Decreto 254/2008, de 1 de agosto, por el que se establece el currículo del Segundo Ciclo de la Educación Infantil en la Comunidad Autónoma de la Región de Murcia (p.182).

1. Conforme a lo establecido en el artículo 7 del Real Decreto 1630/2006, de 29 de diciembre, en el segundo ciclo de la Educación Infantil la evaluación será global, continua y formativa.

2. La observación directa y sistemática constituirá la técnica principal del proceso de evaluación.

3. La evaluación en este ciclo debe servir para identificar los aprendizajes adquiridos y el ritmo y características de la evolución de cada niño o niña. A estos efectos, el tutor, así como el resto de maestros que intervengan en el proceso formativo de los niños, tomarán como referencia los criterios de evaluación de cada una de las áreas.

Por lo tanto, para evaluar la secuencia de enseñanza-aprendizaje Nos movemos por el espacio se va a realizar tres tipos de evaluaciones: una evaluación inicial, una evaluación continua y una evaluación final. 
Una evaluación inicial, donde podamos averiguar los conocimientos previos que tienen los alumnos en torno al tema. De este modo se favorecerá el aprendizaje significativo, ya que relacionaran sus conocimientos previos con la nueva información en las diferentes actividades.

Después una evaluación continua y una evaluación final, ambas para saber que dificultades se nos presentan por el camino, así como para detectar si los objetivos y contenidos que nos habíamos propuesto en la planificación se están cumpliendo. También, la evaluación continua para concienciarnos que aspectos positivos o negativos tenemos que mejorar a lo largo de la puesta en práctica de nuestra secuencia, y tomar decisiones de nuestra labor educativa en relación con la organización de los espacios y los tiempos.

Por último, una evaluación final para reflexionar sobre la práctica educativa para cerciorarnos que se han logrado los objetivos y contenidos que nos hemos planteado al inicio, y para realizar propuestas de mejora si se vuelve a hacer de nuevo otra secuencia.

En referencia con los instrumentos utilizados tal y como indica García-Carmona, Criado y Cañal (2014) para realizar la evaluación de esta secuencia, no solo es preciso centrarse en la observación directa, sino que sería razonable contar con otros instrumentos complementarios:

- Para la evaluación inicial, se hará una entrevista para conocer lo que ya saben los alumnos sobre el tema, para posteriormente introducir los nuevos contenidos que se van a trabajar a lo largo de la secuencia.

- Para la evaluación continua, se colocará un mural en el tablero del aula que se irá completando, a medida que vayan asimilando los nuevos aprendizajes.

- Para la evaluación final, se elaborará una rúbrica en la que se indique cuantos alumnos han conseguido los objetivos, cuantos están en proceso de adquirirlos y cuantos necesitan una ayuda. 
Ilustración 3. Evaluación de la secuencia de enseñanza-aprendizaje Nos movemos por el espacio.

\begin{tabular}{|c|c|c|}
\hline Estándares de aprendizaje & Conseguido $\quad$ En proceso & Necesita ayuda \\
\hline $\begin{array}{c}\text { Interviene activamente en el } \\
\text { patio aportando ideas } \\
\text { previas acerca del espacio. }\end{array}$ & 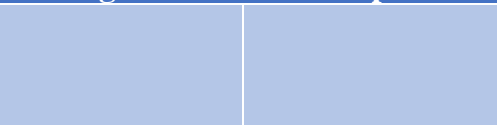 & \\
\hline $\begin{array}{c}\text { Participa en la creación del } \\
\text { carnet de astronauta y } \\
\text { tarjeta de la NASA. } \\
\end{array}$ & & \\
\hline $\begin{array}{c}\text { Conoce y explica algunos } \\
\text { hechos y personas } \\
\text { destacadas en la historia de } \\
\text { la conquista espacial (Neil } \\
\text { Armstrong, Edwin Aldrin } \\
\text { y Michael Collins) a través } \\
\text { del cuento: Apolo XI: Un } \\
\text { viaje a la luna. }\end{array}$ & & \\
\hline $\begin{array}{l}\text { Colabora en la dramatización } \\
\text { del hito histórico: el primer } \\
\text { hombre que piso la Luna. }\end{array}$ & & \\
\hline $\begin{array}{c}\text { Investiga y recoge datos sobre } \\
\text { la fuerza humana, del aire } \\
\text { y del agua: cohetes } \\
\text { propulsados. }\end{array}$ & & \\
\hline $\begin{array}{l}\text { Entiende y ordena las escenas } \\
\text { de la película de Wall-E: } \\
\text { El batallón de limpieza. }\end{array}$ & & \\
\hline \multicolumn{3}{|l|}{$\begin{array}{c}\text { Colabora en la construcción del transporte espacial } \\
\text { recoge basura. }\end{array}$} \\
\hline \multicolumn{3}{|l|}{$\begin{array}{c}\text { Utiliza la fuerza del aire para mover un transporte } \\
\text { espacial recoge basura y resuelve el problema de la } \\
\text { basura espacial por medio del juego Una guerra de } \\
\text { limpieza. }\end{array}$} \\
\hline \multicolumn{3}{|l|}{$\begin{array}{l}\text { Reflexiona colectivamente sobre los conocimientos } \\
\text { adquiridos respecto al espacio y obtiene } \\
\text { conclusiones acerca de lo aprendido en un mural. }\end{array}$} \\
\hline $\begin{array}{l}\text { Aplica lo aprendido a otras situaciones de la vida } \\
\text { mediante el juego Kahoot. }\end{array}$ & & \\
\hline
\end{tabular}




\subsection{REFLEXIÓN SOBRE LA PUESTA EN PRÁCTICA}

Tabla 1 Resultados de evaluación de la secuencia de enseñanza-aprendizaje Nos movemos por el espacio.

\begin{tabular}{|c|c|c|c|}
\hline Criterios de evaluación & Conseguido & En proceso & Necesita ayuda \\
\hline $\begin{array}{c}\text { Interviene activamente en el } \\
\text { patio aportando ideas } \\
\text { previas acerca del espacio. }\end{array}$ & 23 & & 1 \\
\hline $\begin{array}{c}\text { Participa en la creación del } \\
\text { carnet de astronauta y } \\
\text { tarjeta de la NASA. }\end{array}$ & 24 & & \\
\hline $\begin{array}{c}\text { Conoce y explica algunos } \\
\text { hechos y personas } \\
\text { destacadas en la historia de } \\
\text { la conquista espacial (Neil } \\
\text { Armstrong, Edwin Aldrin } \\
\text { y Michael Collins) a través } \\
\text { del cuento: Apolo XI: Un } \\
\text { viaje a la luna. }\end{array}$ & 19 & 5 & \\
\hline $\begin{array}{c}\text { Colabora en la dramatización } \\
\text { del hito histórico: el primer } \\
\text { hombre que piso la Luna. }\end{array}$ & 24 & & \\
\hline $\begin{array}{c}\text { Investiga y recoge datos sobre } \\
\text { la fuerza humana, del aire } \\
\text { y del agua: cohetes } \\
\text { propulsados. }\end{array}$ & 24 & & \\
\hline $\begin{array}{c}\text { Entiende y ordena las escenas } \\
\text { de la película de Wall-E: el } \\
\text { batallón de limpieza. }\end{array}$ & 24 & & \\
\hline $\begin{array}{c}\text { Colabora en la construcción del transporte espacial } \\
\text { recoge basura. }\end{array}$ & 24 & & \\
\hline $\begin{array}{c}\text { Utiliza la fuerza del aire para mover un transporte } \\
\text { espacial recoge basura y resuelve el problema de la } \\
\text { basura espacial por medio del juego Una guerra de } \\
\text { limpieza. }\end{array}$ & 24 & & \\
\hline $\begin{array}{l}\text { Reflexiona colectivamente sobre los conocimientos } \\
\text { adquiridos respecto al espacio y obtiene } \\
\text { conclusiones acerca de lo aprendido en un mural. }\end{array}$ & 22 & & 2 \\
\hline $\begin{array}{l}\text { Aplica lo aprendido a otras situaciones de la vida } \\
\text { mediante el juego Kahoot. }\end{array}$ & 24 & & \\
\hline
\end{tabular}

Tras llevar a cabo la secuencia de enseñanza-aprendizaje, se ha descubierto que los objetivos que se han planificado al inicio se han cumplido de forma satisfactoria. En primer lugar, en la fase de exploración y explicitación inicial, se puede comprobar que 23 alumnos han conseguido intervenir aportando sus conocimientos previos sobre el espacio, excepto una niña que no ha participado por timidez, por lo que necesita ayuda. También, todos han participado en la creación del carnet de astronauta y tarjeta de la NASA. 
En segundo lugar, en la fase de introducción de los nuevos conocimientos, se puede ver en los estándares de aprendizaje que 19 alumnos han conseguido conocer y explicar algunos hechos y personas destacadas en la historia de la conquista espacial a través del cuento: Apolo XI: Un viaje a la luna, pero 5 aún están en proceso de adquirir el estándar de aprendizaje. Sin embargo, todos han colaborado en la dramatización del hito histórico: el primer hombre que piso la Luna. Además han investigado y recogido datos sobre la fuerza; han conocido y ordenado las escenas de la película de Wall-e: El batallón de limpieza; y han construido el transporte espacial recoge basura y competido en el juego Una guerra de limpieza.

De la misma manera, en la fase de estructuración y síntesis de los nuevos conocimientos, se puede observar que 22 alumnos han reflexionado a la misma vez que han obtenido conclusiones de lo aprendido en el mural, pero hay 2 alumnos del aula que todavía no ha conseguido el estándar de aprendizaje.

Por último, en la fase de aplicación, los 24 alumnos han respondido correctamente a las preguntas del juego Kahoot.

Los resultados de posibles pruebas se pueden ver en los Anexos V, VIII, XI y XIV. Y los resultados de claras evidencias se pueden observar en los Anexos III, VI, IX, XII y VX.

\section{CONCLUSIONES, CONSECUENCIAS E IMPLICACIONES EDUCATIVAS}

En conclusión, gracias a que se ha utilizado una metodología como es el juego, ha aportado bastantes beneficios para los alumnos del Segundo Ciclo de la Educación Infantil, ya que les ha motivado y ha partido de sus conocimientos previos, donde han relacionado lo que ya saben con los nuevos aprendizajes. Además, esta metodología ha permitido que los alumnos potencien todas sus facetas de desarrollo (afectivo, social, cognitivo y físico).

Por otro lado, en este trabajo fin de grado se ha podido analizar por primera vez como se planifica y se lleva a la práctica una secuencia de enseñanza-aprendizaje, especialmente de ciencias experimentales. Asimismo, se ha podido restructurar la planificación en algunos casos, añadiendo de esta manera nuevas actividades para cubrir con todos los objetivos que se querían conseguir.

En esta propuesta didáctica, también, se ha podido realizar una evaluación continua, utilizando como instrumento de evaluación los murales, donde se han registrado los aprendizajes que han ido adquiriendo los alumnos durante el desarrollo de la secuencia. Por lo tanto, este tipo de evaluación ha resultado ser bastante gratificante, puesto que ha ayudado en gran medida al alumnado a recordar, reflexionar y obtener conclusiones sobre los contenidos aprendidos.

Una situación que pareció bastante sorprendente fue cuando en la actividad 4 se puso una película de media hora, con el único objetivo de concienciar al alumnado del problema de la basura espacial. Pero tras introducir el juego de Una guerra de limpieza, en el que se les hacía participe de manera directa a los 
alumnos, se pudo comprobar como las expectativas negativas que se tenían sobre la actividad convirtieron esos objetivos perseguidos en aprendizajes significativos para los alumnos.

En el caso de planificar de nuevo la misma secuencia de enseñanza, se podría comprar el cuento Apolo XI: Un viaje a la luna para enseñar los materiales con mejor calidad y poder conservarlos más tiempo. Y en segundo lugar, quitar la película de Wall-e: El batallón de limpieza, ya que su duración es demasiado larga como para mantener atentos a todo el público infantil, y mostrar solo escenas que sean importantes del visionado.

No obstante, las actividades que se podrían plantear de nuevo son la dramatización, Investigamos ¿Cómo puede moverse un cohete?, el juego Guerra de limpieza y el Kahoot. Todas estas actividades porque han motivado al alumnado y los han metido de lleno en cada una de las explicaciones.

Una de las fortalezas de este Trabajo Fin de Grado ha sido que algunos de los recursos que han ido fabricando los alumnos, como por ejemplo transportes recoge-basura se han aprovechado para el proyecto del aula $E l$ Espacio. Sin embargo, una de las debilidades ha sido que la mayoría de las actividades se han llevado a cabo en gran grupo y de forma individual, aunque hubiese estado bien utilizar una metodología como es el aprendizaje cooperativo. 


\section{BIBLIOGRAFÍA}

Baños, I. B. (2018). Tema 3. La materia, los materiales, los objetos [diapositivas de Power Point].

Cantó, J., de Pro, A. y Solbes, J. (2016). ¿Qué ciencias se enseñan y cómo se hace en las aulas de educación infantil? La visión de los maestros en formación inicial. Enseñanza de las ciencias, 34(3), 25-50. Recuperado de https://www.raco.cat/index.php/Ensenanza/article/view/314144

Decreto No. 254/2008. Decreto por el que se establece el currículo del Segundo Ciclo de la Educación Infantil en la Comunidad Autónoma de la Región de Murcia. Publicada en Boletín Oficial de la Región de Murcia No. 182, del 6 de agosto de 2008. España.

Fernández Oliveras, A. y García Pete, L. C. (2015). Juego, Educación Infantil y Ciencias Experimentales en la Literatura Educativa. REIDOCREA, 4(1), 1-12. Recuperado de http://digibug.ugr.es/bitstream/handle/10481/37109/FernandezOliveras_LiteraturaEducativa.pdf?sequence=6\&is Allowed=y

Gallego Madrid, D. E., Quinceno Serna, Y. y Pulgarín Vásquez, D. (2014). Unidades didácticas: Un camino para la transformación de la enseñanza de las ciencias desde un enfoque investigativo. Revista Tecné, Episteme y Didaxis: TED, (extraordinario), 923-934. doi: 10.17227/01203916.3460

García-Carmona, A.; Criado, A. M. y Cañal, P. (2014). Alfabetización científica en la etapa 3-6 años: un análisis de la regulación estatal de enseñanzas mínimas. Enseñanza de las Ciencias, 32(2), 131-149. https://doi.org/10.5565/rev/ensciencias.817

Mazas, B., Gil-Quílez, M. J., Martínez-Peña, B., Hervás, A., \& Muñoz, A. (2018). Los niños de infantil piensan, actúan y hablan sobre el comportamiento del aire y del agua. Enseñanza de las ciencias, 36(1), 163-180. Recuperado de https://www.raco.cat/index.php/Ensenanza/article/view/335279

Melo Herrera, M. P. y Hernández Barbosa, R. (2014). El juego y sus posibilidades en la enseñanza de las ciencias naturales. Innovación Educativa, 14(66), 41-64. Recuperado de http://www.scielo.org.mx/pdf/ie/v14n66/v14n66a4.pdf

Morales Ruiz, J.J (2015). Maria Montessori y la educación cósmica, REHMLAC+, 7(2), 201-237. doi: 10. 15517/rehmlac.v712.22697

Rodríguez Palmero, M. (2011). La teoría del aprendizaje significativo: una revisión aplicable a la escuela actual. IN. Revista Electrònica d'investigació i Innovació Educativa i Socioeducativa, 3(1), 29-50. Recuperado de https://dialnet.unirioja.es/servlet/articulo?codigo=3634413

Sanchidrián Blanco, C., Ruiz Berrio, J., Colmenar Orzaes, C., Diego Pérez, C., Egido Gálvez, I., García Crespo, C.,... Vera Vila, J. (Ed.). (2010). Historia y perspectiva actual de la educación infantil. Barcelona, España: Editorial GRAÓ.

Spektor-Levy, O., Kesner Baruch, Y. y Mevarech, Z. (2013). Science and Scientific Curiosity in Pre-school. The teacher's point of view. International Journal of Science Education, 35(13), 2.226-2.253. doi: 10.1080/09500693.2011.631608

Varela, M., Pérez, U., Ulla, A., y Arias, A. (2012). Problemáticas del proceso de enseñanza y aprendizaje de la astronomía. Boletín das ciencias, 25(76), 107-109.

Vila, J. y Sierra, C. J. (2008). Explicación con experimentos sencillos y al alcance de todos de la primera ley de Newton (la ley de la inercia) así como la diferencia entre inercia e inercialidad. Lat. Am. J. Phys. Educ, 2(3), 241245. Recuperado de https://dialnet.unirioja.es/servlet/articulo?codigo=2734653 


\section{ANEXOS}

ANEXO 1

Tabla 2 Objetivos curriculares y específicos de la secuencia por orden de relevancia.

\section{ÁREA 2. CONOCIMIENTO DEL ENTORNO}

\section{Objetivos del currículo}

Observar y explorar de forma activa su entorno, generando interpretaciones sobre algunas situaciones y

hechos significativos y mostrando interés por su conocimiento.

\section{Objetivos específicos}

Investigar y recoger datos sobre la fuerza humana, del aire y del agua: cohetes propulsados.

Entender y ordenar las escenas de la película de WallE: el batallón de limpieza. funcionalmente elementos y colecciones, identificando sus atributos y cualidades, y estableciendo relaciones de agrupamientos, clasificación, orden y cuantificación.

Identificar y representar relaciones espaciales y geométricas.

Conocer y valorar los componentes básicos del medio natural y algunas de sus relaciones, cambios y transformaciones, desarrollando actitudes de cuidado, respeto y responsabilidad en su conservación.
Investigar y recoger datos sobre la fuerza humana, del aire y del agua: cohetes propulsados.

Utilizar la fuerza del aire para mover un transporte espacial recoge basura y resolver el problema de la basura espacial por medio del juego Una guerra de limpieza.

\section{ÁREA 3. LENGUAJES: COMUNICACIÓN Y REPRESENTACIÓN}

\section{Objetivos del currículo}

Utilizar la lengua como instrumento de comunicación funcional, de representación, aprendizaje y disfrute, de expresión de ideas y sentimientos, y valorar la lengua oral como un medio de relación con los demás y de regulación de la convivencia.

Comprender, reproducir y recrear algunos textos literarios y de tradición cultural propios de nuestra Comunidad, mostrando actitudes de valoración, disfrute e interés hacia ellos.

Acercarse al conocimiento de obras artísticas expresadas en distintos lenguajes y realizar actividades de representación y expresión artística mediante el empleo de diversas técnicas.

\section{Objetivos específicos}

Intervenir activamente en el patio aportando ideas previas acerca del espacio.

Reflexionar colectivamente sobre los conocimientos adquiridos respecto al espacio y obtener conclusiones acerca de lo aprendido en un mural.

Colaborar en la dramatización del hito histórico: el primer hombre que piso la Luna.

Entender y ordenar la película de Wall-E: el batallón de limpieza.

Conocer y explicar algunos hechos y personas destacadas en la historia de la conquista espacial (Neil Armstrong, Edwin Aldrin y Michael Collins) a través del cuento: Apolo XI: Un viaje a la luna

Participar en la creación del carnet de astronauta y tarjeta de la NASA.

Colaborar en la construcción del transporte espacial recoge basura.

\section{ÁREA 1. CONOCIMIENTO DE SÍ MISMO Y AUTONOMÍA PERSONAL}

\section{Objetivos del currículo}

Participar en juegos colectivos respetando las reglas establecidas y valorar el juego como medio de relación social y recurso de ocio y tiempo libre.

\section{Objetivos específicos}

Utilizar la fuerza del aire para mover un transporte espacial recoge basura y resolver el problema de la basura espacial por medio del juego Una guerra de limpieza.

Aplicar lo aprendido a otras situaciones de la vida mediante el juego Kahoot 
Tabla 3 Contenidos curriculares y específicos de la secuencia de manera secuenciada.

\section{ÁREA 1. CONOCIMIENTO DE SÍ MISMO Y AUTONOMÍA PERSONAL}

\section{Contenidos del currículo}

BLOQUE 2. Juego y movimiento

Confianza en las propias posibilidades de acción, participación y esfuerzo personal en los juegos y en el ejercicio físico. Gusto por el juego.

\section{Contenidos específicos}

BLOQUE 2. Juego y movimiento

Utilización de la fuerza del aire para mover un transporte espacial recoge basura y resolver el problema de la basura espacial por medio del juego Una guerra de limpieza.

Aplicación de lo aprendido a otras situaciones de la vida mediante el juego Kahoot.

\section{ÁREA 2. CONOCIMIENTO DEL ENTORNO \\ Contenidos del currículo \\ BLOQUE 1. Medio físico: elementos, relaciones y medida \\ Los objetos y materias presentes en el medio, sus funciones y usos cotidianos. Interés por su exploración y actitud de respeto y cuidado hacia objetos propios y ajenos. \\ Establecimiento de relaciones de agrupamiento de elementos y colecciones. Interés por la clasificación de elementos y por explorar sus cualidades y grados. \\ Contenidos específicos \\ BLOQUE 1. Medio físico: elementos, relaciones y medida \\ Indagación y recogida de datos sobre la fuerza \\ humana, del aire y del agua: cohetes propulsados \\ Comprensión y secuenciación de las escenas de la película de Wall-E: el batallón de limpieza. \\ Establecimiento de relaciones sencillas entre los materiales que constituyen los objetos, su comportamiento (rodar, caer, botar, arrastrar, flotar, ser atraídos por un imán, proyectar sombras...) y su utilización en la vida cotidiana. \\ Indagación y recogida de datos sobre la fuerza humana, del aire y del agua: cohetes propulsados. \\ Utilización de la fuerza del aire para mover un transporte espacial recoge basura y resolver el problema de la basura espacial por medio del juego Una guerra de limpieza.}

BLOQUE 2. Acercamiento a la naturaleza Participación activa en la resolución de problemas medioambientales. Recogida selectiva de residuos, limpieza de espacios en su entorno físico próximo.
BLOQUE 2. Acercamiento a la naturaleza Implicación en la construcción del transporte espacial recoge basura.

Utilización de la fuerza del aire para mover un transporte espacial recoge basura y resolver el problema de la basura espacial por medio del juego Una guerra de limpieza.

\begin{tabular}{|c|c|}
\hline \multicolumn{2}{|c|}{ ÁREA 3. LENGUAJE: COMUNICACIÓN Y REPRESENTACIÓN } \\
\hline Contenidos del currículo & Contenidos específicos \\
\hline BLOQUE 1. Lenguaje verbal & BLOQUE 1. Lenguaje verbal \\
\hline Escuchar, hablar y conversar & Escuchar, hablar y conversar \\
\hline $\begin{array}{c}\text { Utilización y valoración progresiva de la lengua oral } \\
\text { para evocar y relatar hechos, para explorar }\end{array}$ & $\begin{array}{l}\text { Conocimientos previos sobre el espacio. Formulación } \\
\text { de hipótesis y expresión de opiniones e ideas. }\end{array}$ \\
\hline $\begin{array}{c}\text { sentimientos y como ayuda para regular la propia } \\
\text { conducta y la de los demás. }\end{array}$ & $\begin{array}{c}\text { Razonamiento en colectivo sobre los conocimientos } \\
\text { adquiridos respecto al espacio y obtener conclusiones } \\
\text { acerca de lo aprendido en un mural. }\end{array}$ \\
\hline Acercamiento a la literatura & Acercamiento a la literatura \\
\hline $\begin{array}{l}\text { Escucha y comprensión de cuentos, tanto tradicionales } \\
\text { como contemporáneas, como fuente de placer y de } \\
\text { aprendizaje en su lengua materna. }\end{array}$ & $\begin{array}{c}\text { Aprendizaje y explicación de algunos hechos y } \\
\text { personas destacadas en la historia de la conquista } \\
\text { espacial (Neil Armstrong, Edwin Aldrin y Michael } \\
\text { Collins) a través del cuento: Apolo XI: Un viaje a la } \\
\text { luna }\end{array}$ \\
\hline
\end{tabular}


BLOQUE 2. Lenguaje audiovisual y tecnologías de la $\quad$ BLOQUE 2. Lenguaje audiovisual y tecnologías de la información y la comunicación información y la comunicación

Acercamiento a producciones audiovisuales como películas, dibujos animados o videojuegos que ayuden

a la comprensión de contenidos educativos.

Valoración crítica de sus contenidos y de su estética.

Comprensión y secuenciación de las escenas de la película de Wall-E: el batallón de limpieza.

Aplicación de lo aprendido a otras situaciones de la vida mediante el juego Kahoot.

BLOQUE 3. Lenguaje artístico Expresión plástica

Participación en realizaciones colectivas. Interés y respeto por las elaboraciones plásticas propias y de los demás.

\section{BLOQUE 3. Lenguaje artístico} Expresión plástica

Expresión de la originalidad y la creatividad en el proceso de creación del carnet de astronauta y tarjeta de la NASA.

Implicación en la construcción del transporte espacial recoge basura.

BLOQUE 4. Lenguaje corporal

Participación en actividades de dramatización, danzas, bailes, juego simbólico y otros juegos de expresión corporal. Interés e iniciativa para participar en

\section{BLOQUE 4. Lenguaje corporal}

Participación en la dramatización del hito histórico: el primer hombre que piso la Luna representaciones.

\section{ANEXO II}

Ilustración 4. Preguntas para detectar las ideas previas de los alumnos de 5 años A sobre el espacio.

¿Qué vemos?

¿Cómo será?

¿Podemos visitarlo?

¿Qué necesitamos para viajar hasta allí (señalando el cielo)?

¿Y cómo puede moverse un cohete?

¿Qué personas son las que viajan continuamente al espacio?

¿Cómo se tienen que vestir?

¿Qué pasa si no se disfrazan de astronautas?

¿Pensáis que en el espacio se tira basura?

¿Alguien podría decir un ejemplo de esa basura? 


\title{
ANEXO III
}

Ilustración 5. Respuestas donde se detectan las ideas previas de los alumnos 5 años A sobre el espacio

\author{
¿Qué vemos? El cielo (Alumno \\ $1,2,3,4,5,6,7,8,9,10,11,12,13,14,15,16,17,18,19,20,21$, \\ $22,23)$ \\ ¿Cómo será? De color azul como el cielo. (Alumno \\ $1,2,3,4,5,6,7,8,9,10,11,12,13,14,15,16,17,18,19,20,21$, \\ $22,23)$ \\ ¿Podemos visitarlo? Sí (todos) \\ ¿Qué necesitamos para viajar hasta allí (señalando el \\ cielo)? Necesitamos un cohete para viajar allí. (Alumno \\ $1,2,3,4,5,6,7,8,9,10,11,12,13,14,15,16,17,18,19,20,21$, \\ $22,23)$
}

¿Y cómo puede moverse un cohete? Puede moverse con fuego. (Alumno 1,2,3,4,5,6,7,8,9,10,11,12,13,14,15,16,17, $18,19,20,21,22,23)$

¿Qué personas son las que viajan continuamente al espacio? Los astronautas (todos)

¿Cómo se tienen que vestir? Tienen que ponerse un traje que pesa mucho de color blanco. (Alumno 21)

¿Qué pasa si no se disfrazan de astronautas? Que no pueden respirar y se pueden morir (Alumno $1,2,3,4,5,6,7,8,9,10,11,12,13,14,15,16,17,18,19,20,21$, $22,23)$

¿Pensáis que en el espacio se tira basura? No (Alumno $1,2,3,4,5,6,7,8,9,10,11,12,13,14,15,16,17,18,19,20,21$, 22). Sí (Alumno 23)

¿Alguien podría decir un ejemplo de esa basura?

Cuando un cohete se rompe en el Espacio (Alumno 23)

\section{ANEXO IV}

Ilustración 6. Ficha del carnet de astronauta.

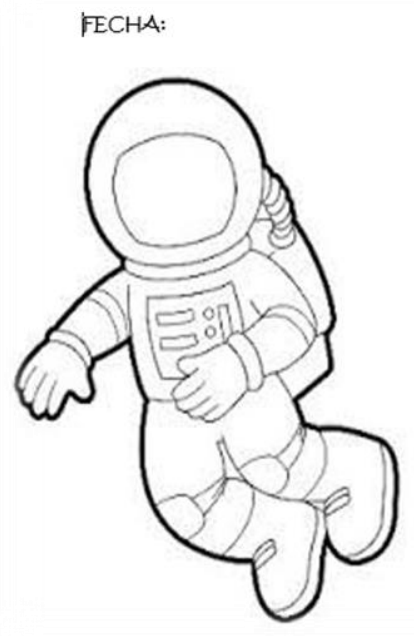

CARNET DE ASTRONAUTA

NOMBRE:

VIAJE A:

MI COHETE SE LLAMA:

¿QUÉ HE HECHO EN LA LUNA?:

FIRMA: 
Ilustración 7. Logotipo de la NASA

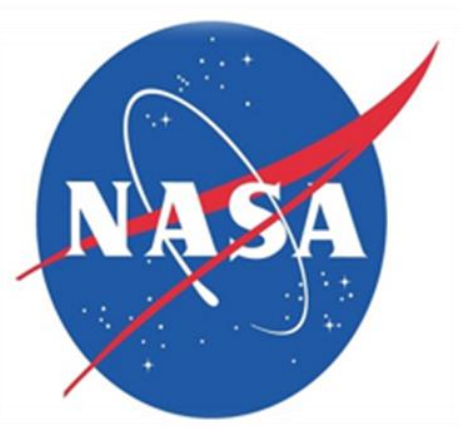

Ilustración 8. Pinzas de colores.

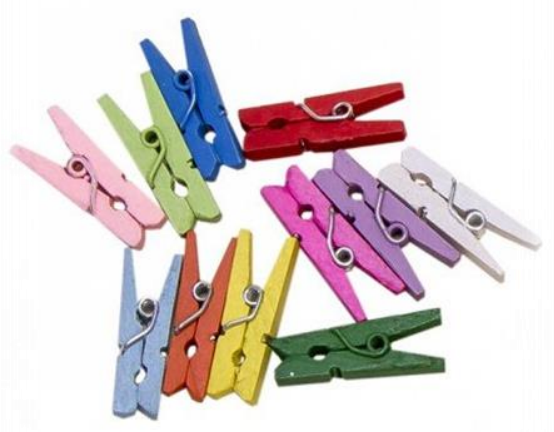

ANEXO V

Ilustración 9. Proceso de creación del carnet de astronauta.

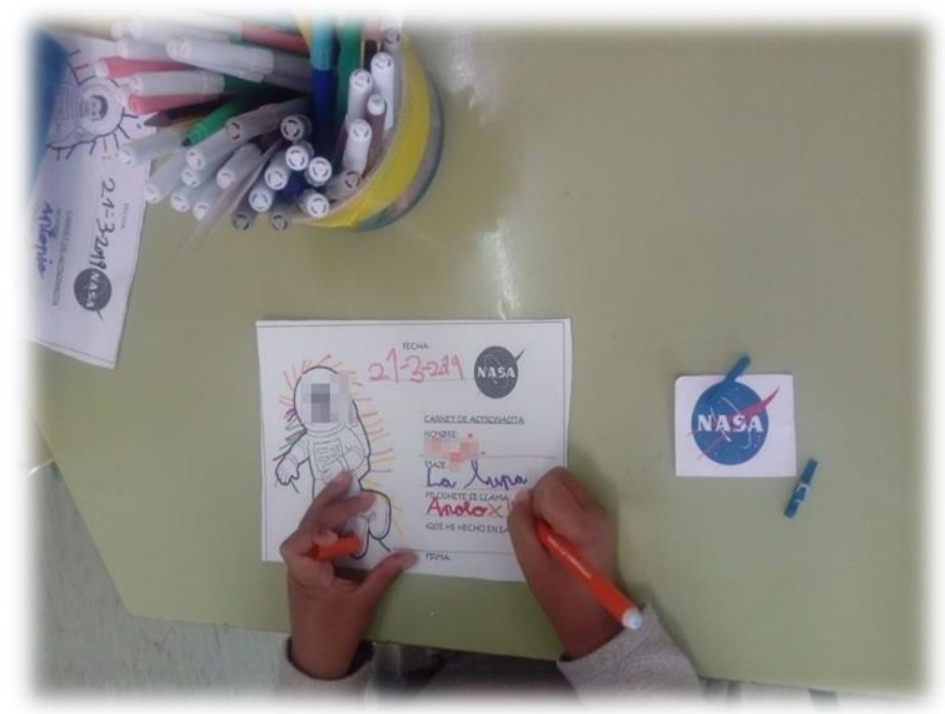


Ilustración 10. Proceso de creación del carnet de astronauta

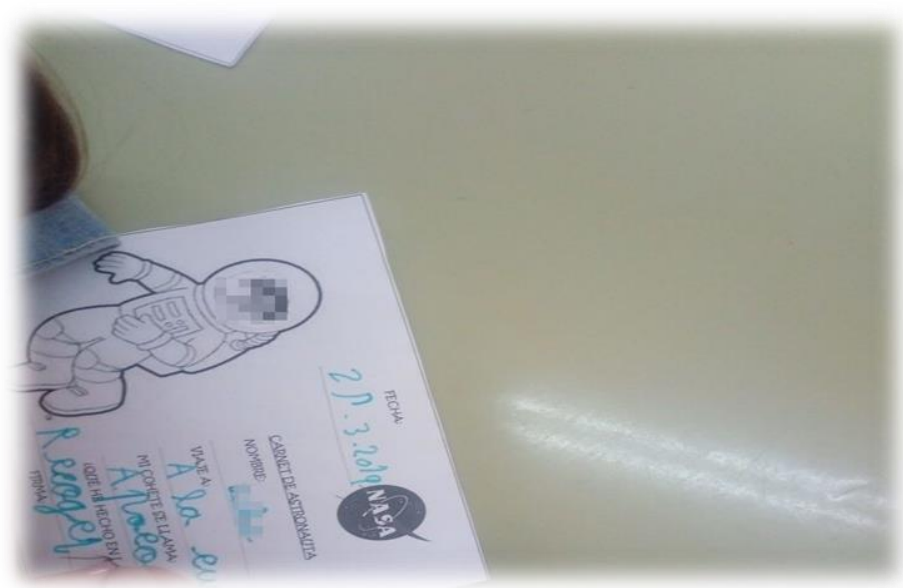

ANEXO VI

Ilustración 11. Elaboración de las tarjetas con el logotipo de la NASA.

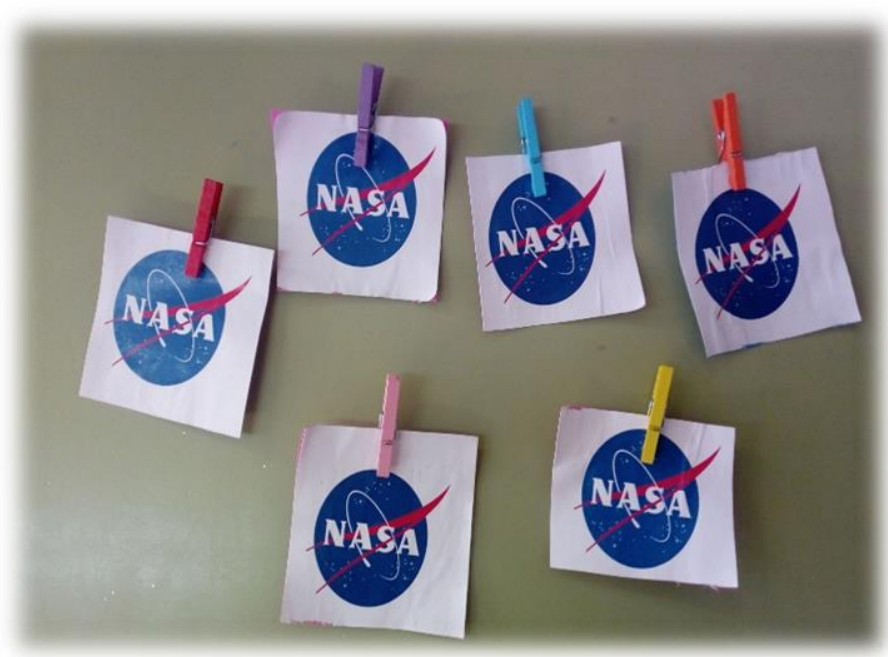

Ilustración 12. Creación del carnet de astronauta.

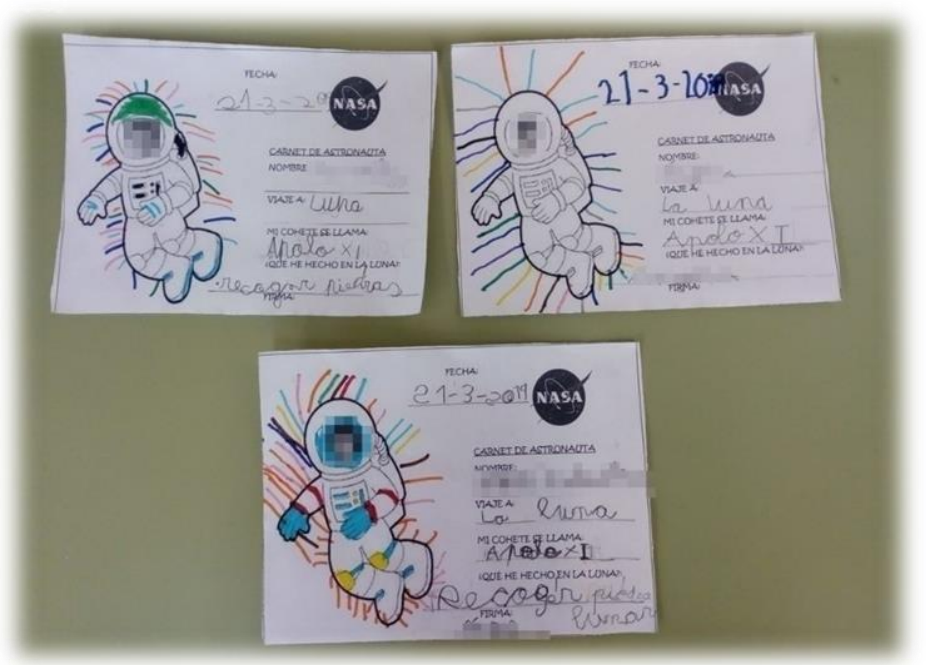


Ilustración 13. Cuento "Apolo XI: Un viaje a la Luna"

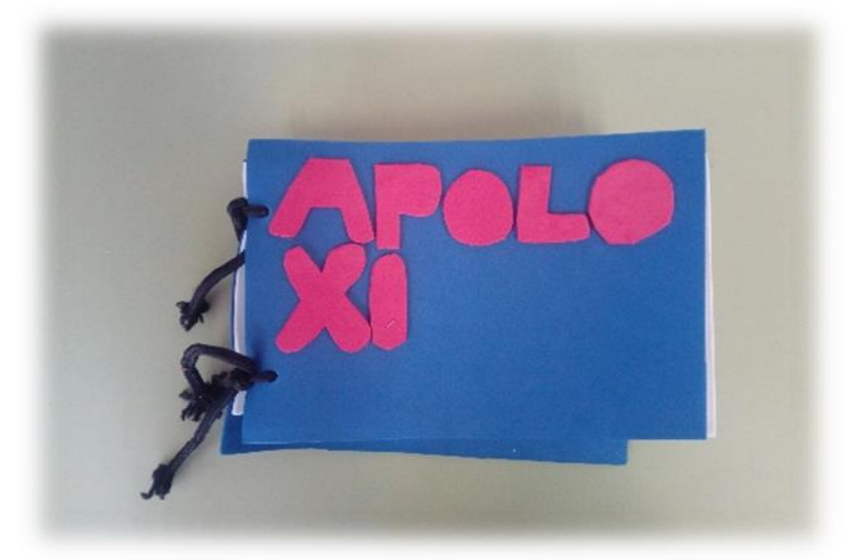

Ilustración 14. Preguntas del cuento "Apolo XI: Un viaje a la Luna" ¿A dónde quería viajar el hombre?

¿Quién era el primer ser vivo que viajó al Espacio?

¿Cómo se llamaba la misión?

¿Qué nave había que construir para aterrizar en la Luna en aquella época?

¿Cómo se llamaba la nave?

¿De qué tanques estaba llena la nave?

¿Qué se había conseguido al principio de esta misión?

¿Qué le propusieron a los astronautas en el segundo momento de la misión?

¿Por qué tenían que tener mucho cuidado en el momento del alunizaje?

Ilustración 25. Preguntas del cuento "Apolo XI: Un viaje a la Luna" ¿Quién tomó el mando de la nave y alunizó?

¿Y entonces quién fue el primer hombre que pisó la Luna? ¿Cuál fue su frase?

¿Qué recogieron Aldrin y Neil Armstrong?

¿Y qué colocaron en la superficie lunar antes de marcharse?

¿De regreso a la Tierra en qué lugar descendieron?

¿Con qué lo hicieron?

¿Finalmente llegaron sanos y salvos los tres astronautas que emprendieron la misión Apolo XI? 
Ilustración 163. Traje completo de astronauta.

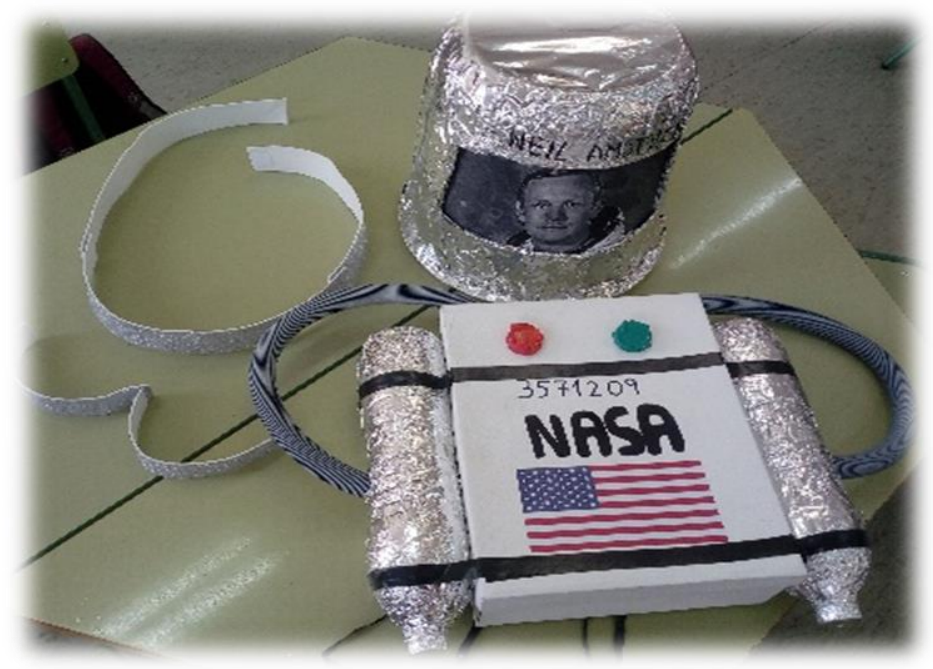

Elaboración propia.

Ilustración 47. Bandera de Estados Unidos.

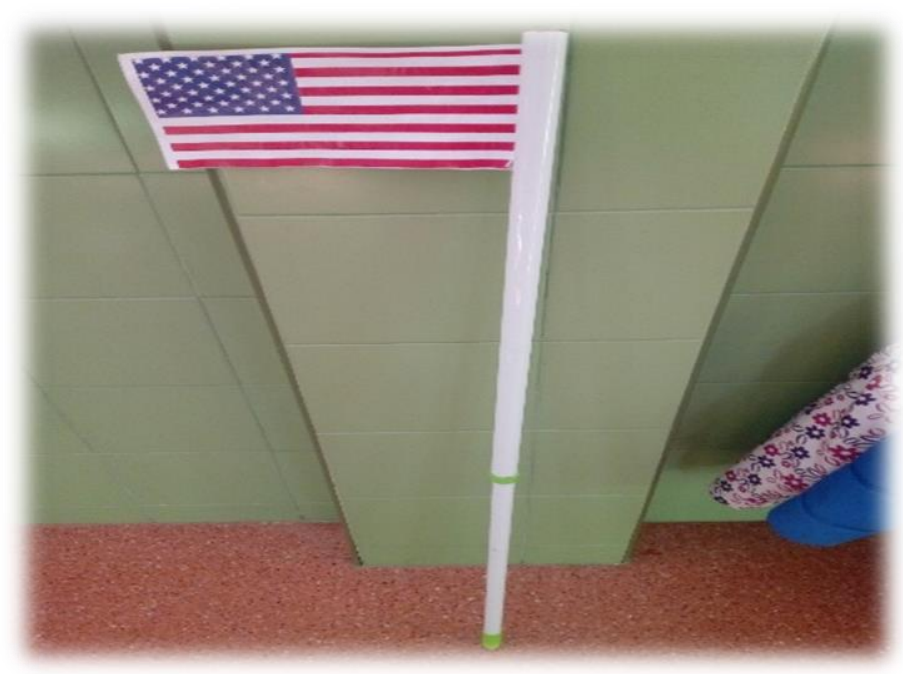

Elaboración propia.

Dramatización "Apolo XI: Un viaje a la Luna”

Neil Armstrong: (La maestra saluda a los alumnos y se presenta). Hola compañeros me llamo Neil Armstrong y soy un astronauta muy ocupado. Quiero viajar a la luna y no sé con qué vehículo ir ¿me ayudáis? (los alumnos responderán sí)

Neil Armstrong: Bien, entonces ¿creo que iré en bicicleta? ¿o puedo ir en moto? Ah vale tengo una idea mejor iré en coche (los niños intervendrán y dirán que tiene que ir en cohete)

Neil Armstrong: Vamos, vamos, vamos subamos al cohete (mover las manos hacia un lado y hacia el otro indicando ir hacia un punto concreto). Ups, creo que se nos olvida una cosa importantísima, nuestra vestimenta. Podemos ponernos un chándal quizás o un vaquero y una camiseta. ¿Qué pensáis? (los alumnos volverán a participar y responderán que se necesita un traje de astronauta) 
Neil Armstrong: Me molesta y me pesa mucho el casco (cruzo los dos brazos simulando que estoy triste). No quiero ponérmelo (moviendo a la derecha y a la izquierda el dedo). Seguro que no pasara nada, podre ir al espacio y volar y volar y volar, ver otros planetas(los niños hablaran de nuevo y dirán que el casco es esencial llevarlo porque sin él no puedes respirar dentro del espacio)

Neil Armstrong: (abrir la boca interpretando que estas sorprendida) Dejemos las preguntas y subamos de una vez al cohete (hacer el gesto de subir una pierna y luego la otra simulando que estas montando en un vehículo) para llevar a cabo nuestra misión que se llama Apolo XI (la maestra indicará que repitan el nombre de la misión varias veces hasta que se familiaricen con ella)

Neil Armstrong: Entre todos tenemos que girar la palanca hacia delante para despegar el cohete ¿me ayudáis? (los alumnos se juntaran en piña y moverán la mano hacia delante tratando de poner en funcionamiento el cohete).

Neil Armstrong: En 10 segundos despegamos. Todos a sus asientos. Nos abrochamos los cinturones. (los niños se sentarán y harán el gesto de abrocharse los cinturones)

Neil Armstrong: 10, 9, 8, 7, 6, 5, 4, 3, 2, 1. ¡DESPEGUE!

Neil Armstrong: (Gesto de sorprendido) Llegamos al espacio. Mirar por la ventana. No me lo creo el cielo es de otro color, un color más oscuro.

Neil Armstrong: Cuidado nos agachamos que nos chocamos con un cohete, giramos a derecha que se acerca otro y ahora a la izquierda (mover la cabeza hacia arriba, a la derecha y a la izquierda respectivamente). Ahora nos inclinamos. Pero sí todo esto es basura espacial ¡uffff! menos mal que estamos a salvo que susto. (Cara de asustado)

Neil Armstrong: Chicos de lejos se puede ver el Sol, que brillante y el planeta Marte. Ahora me tenéis que ayudar a buscar por la ventana la luna (poner las dos manos en la cabeza simulando que está asomándose). El primero que la encuentre grita LUNA.

Neil Armstrong: (Aplaudimos) iiiAterrizamos con éxito!!! Tenemos que bajar del cohete poco a poco sin caernos. Podemos observar la luna de cerca y recoger datos de cómo es verdaderamente (su forma, el tamaño y su textura) y llevarnos una piedra lunar cada uno para tener un recuerdo de nuestro viaje.

Neil Armstrong: Ahora clavamos la bandera (un niño coge la bandera de estados unidos y la clava en la luna). ¡Que bien! Somos los primeros astronautas en pisar la luna. Una fotografía para recordar este momento histórico.

Neil Armstrong: ¡OH NO NO NO! Nos quedamos sin oxígeno si estamos mucho tiempo fuera en el espacio.

Neil Armstrong: Subamos al cohete nos vamos de vuelta a la Tierra para contar este acontecimiento. Lo ponemos en marcha de nuevo ¿me ayudáis? En 10 segundos despegamos. Todos a sus 
asientos. Nos abrochamos los cinturones. (los niños se sentarán y harán el gesto de abrocharse los cinturones)

Neil Armstrong: 10, 9, 8, 7, 6, 5, 4, 3, 2, 1. ¡DESPEGUE!

Neil Armstrong: Regresamos por fin a la Tierra.

Neil Armstrong ¿Os ha gustado el viaje? A mí me ha encantado me lo he pasado genial. Adiós chicos hasta la próxima misión de Apolo XII.

ANEXO VIII

Ilustración 18. Dramatización “Apolo XI: Un viaje a la Luna”.

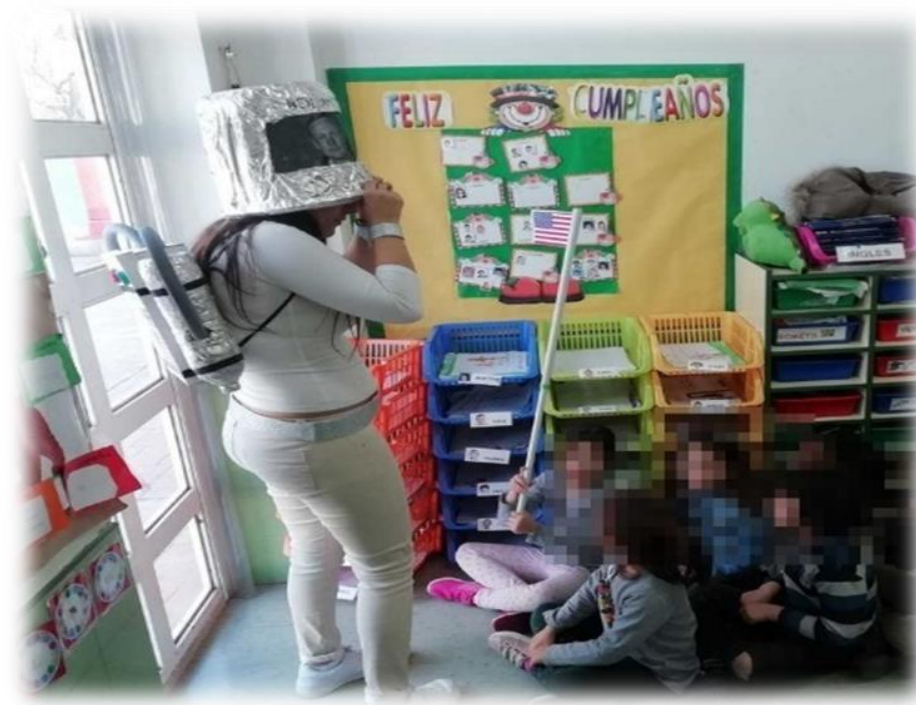

Ilustración 19. Dramatización “Apolo XI: Un viaje a la Luna”.

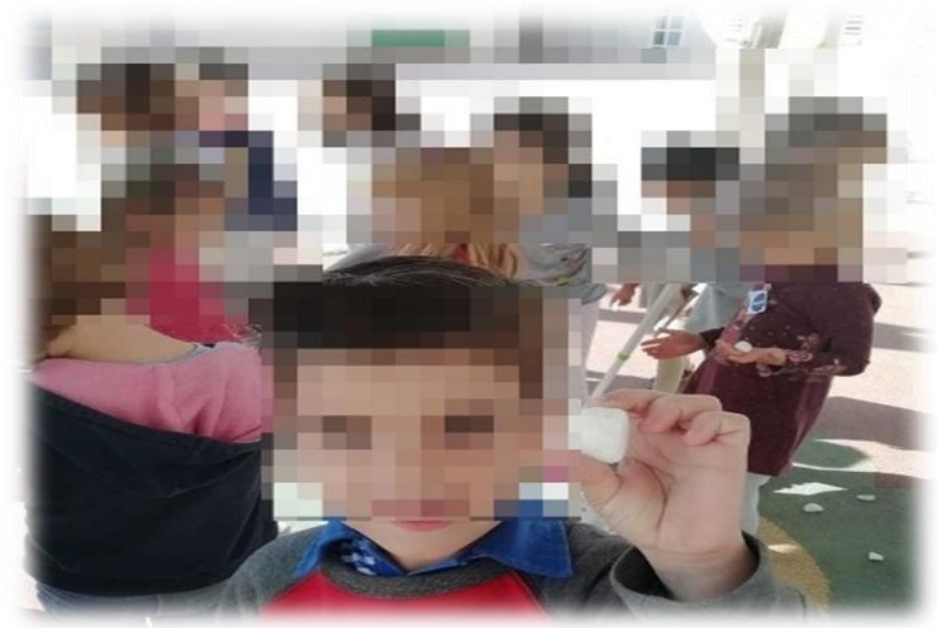




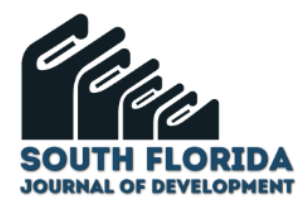

Ilustración 20. Dramatización “Apolo XI: Un viaje a la Luna”.

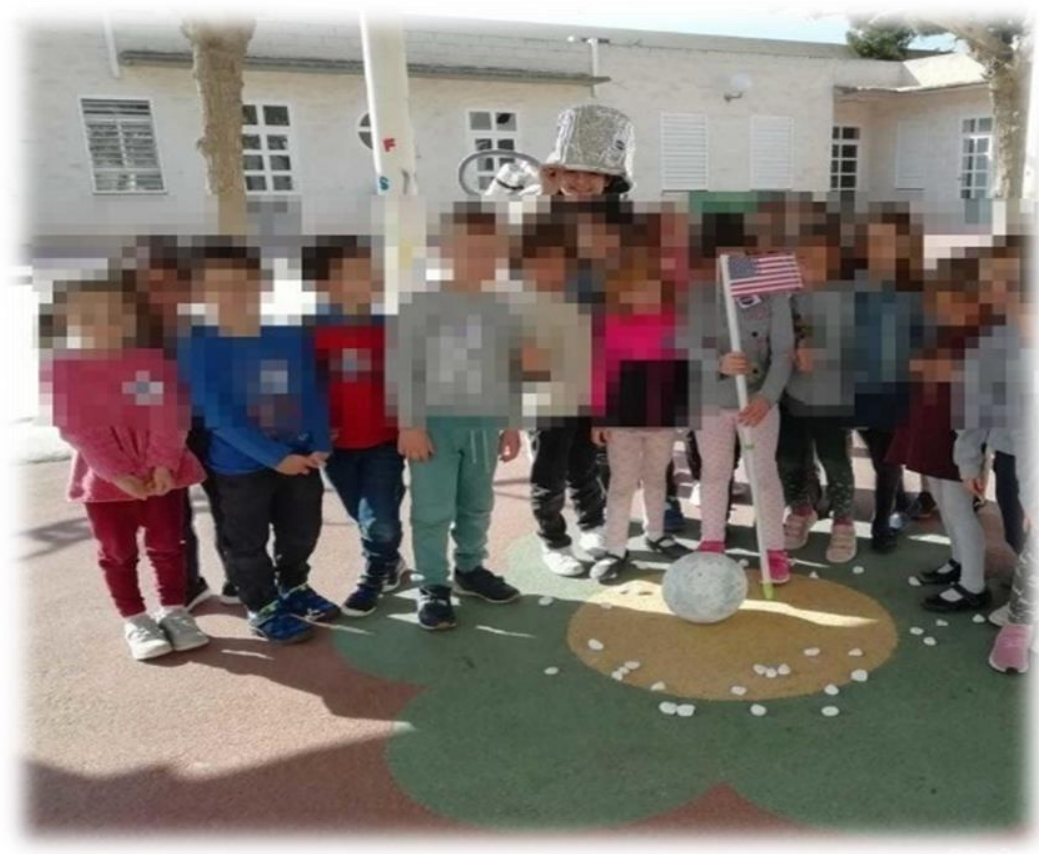

Ilustración 21. Dramatización “Apolo XI: Un viaje a la Luna”.

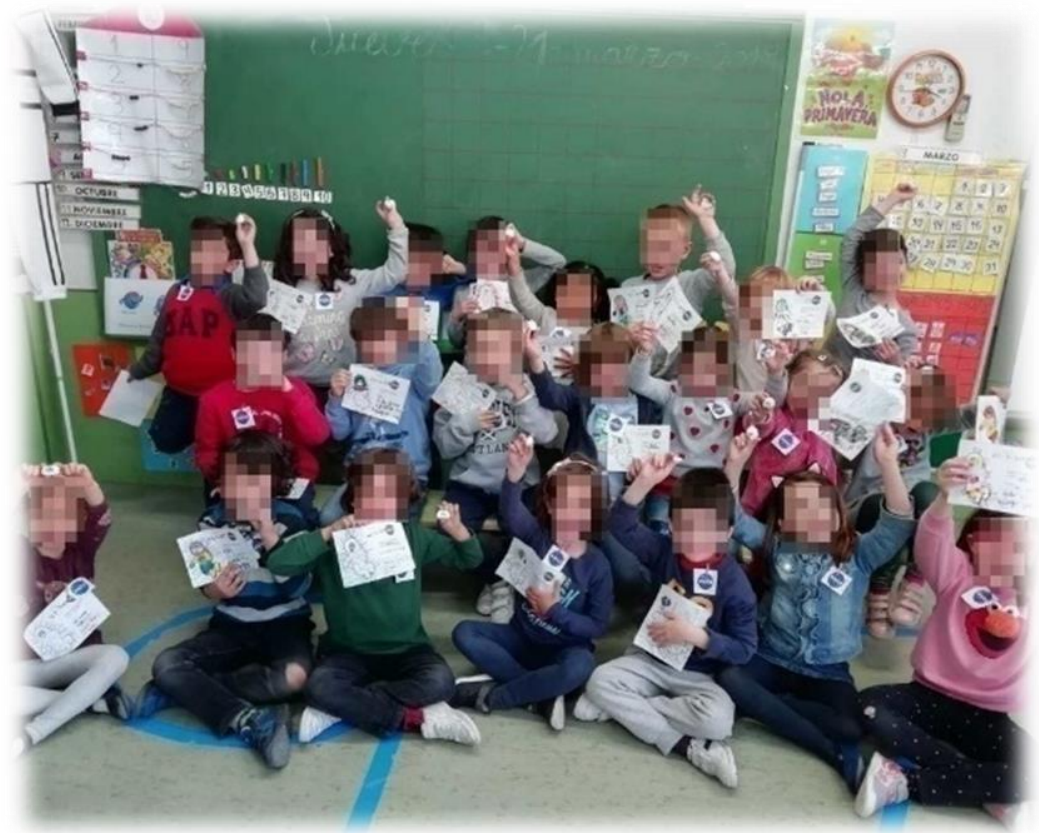


ANEXO IX

Ilustración 5. Respuestas del cuento "Apolo XI: Un viaje a la Luna". ¿A dónde quería viajar el hombre? A la Luna. (todos)

¿Quién era el primer ser vivo que viajó al Espacio? La perra Laika. (todos)

¿Cómo se llamaba la misión? Apolo XI. (todos)

¿Qué nave había que construir para aterrizar en la Luna en aquella época? Una nave con mucha potencia. (Alumno $10,11$ y 12$)$

¿Cómo se llamaba la nave? Saturn V. (todos)

¿De qué tanques estaba llena la nave? De Fuego. (todos)

¿Qué se había conseguido al principio de esta misión?

Despegar la nave al espacio. (Alumno 9)

¿Qué le propusieron a los astronautas en el segundo momento de la misión? Aterrizar en la Luna. (todos)

¿Por qué tenían que tener mucho cuidado en el momento del alunizaje? Porque podían chocar con la Luna. (Alumno 2)

Ilustración 63. Respuestas del cuento "Apolo XI: Un viaje a la Luna". ¿Quién tomó el mando de la nave y alunizó? Neil Armstrong. (todos)

¿Y entonces quién fue el primer hombre que pisó la Luna? ¿Cuál fue su frase? Un pequeño paso para el hombre, un gran salto para la humanidad. (todos)

¿Qué recogieron Aldrin y Neil Armstrong? Pruebas de la luna. (Alumno 6)

¿Y qué colocaron en la superficie lunar antes de marcharse? La bandera de Estados Unidos. (Alumno 15)

¿De regreso a la Tierra en qué lugar descendieron? En un lugar donde había una playa. (Alumno 18)

¿Con qué lo hicieron? Con un paracaídas. (Alumno 6)

¿Finalmente llegaron sanos y salvos los tres astronautas que emprendieron la misión Apolo XI? Sí. (todos) 
Ilustración 7. Piedras lunares con las iniciales de los alumnos.

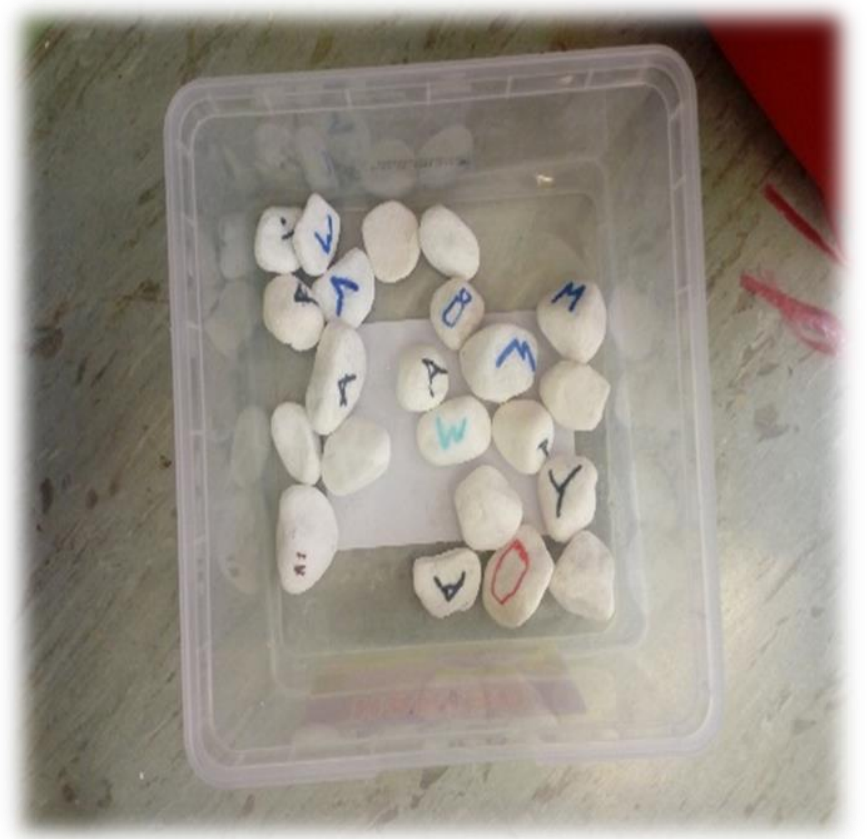

ANEXO X

Ilustración 24. Ficha de la investigación de la fuerza humana, la fuerza del aire y la fuerza del agua.

Nombre:

Experimento: ¿Cómo puedo mover un cohete?

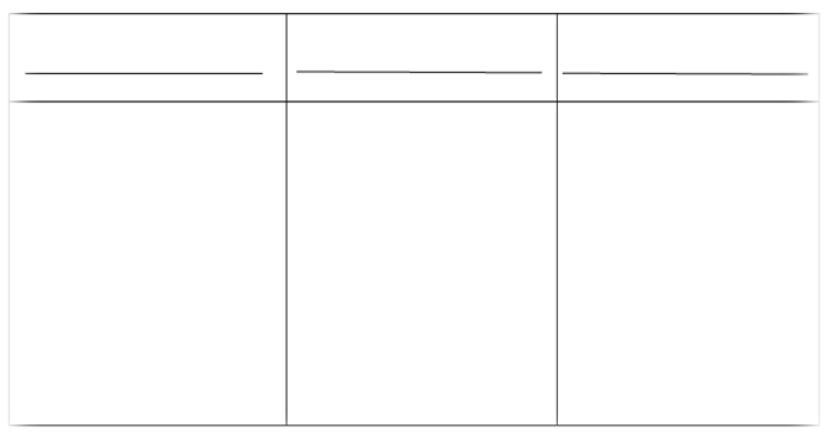


ANEXO XI

Ilustración 85. Investigación de la fuerza humana.

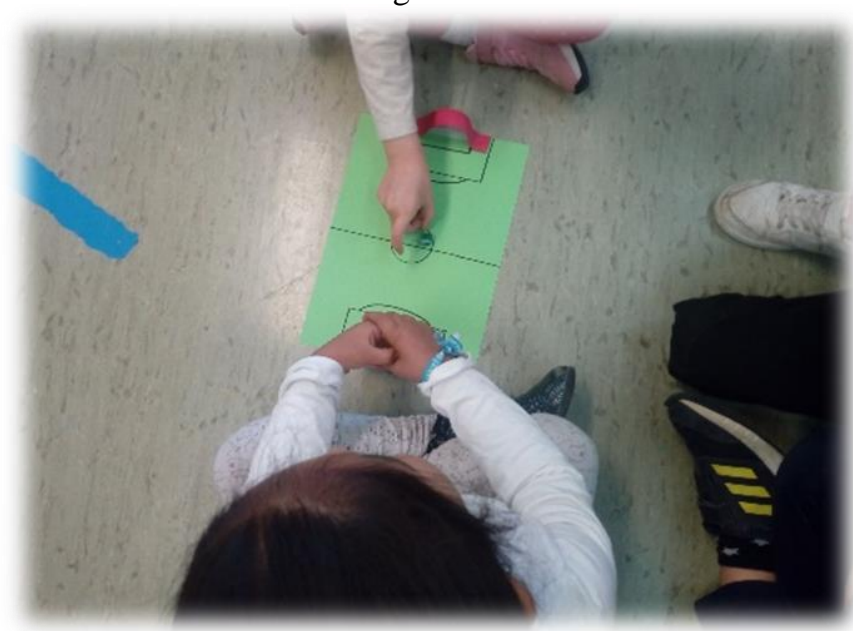

Ilustración 26. Investigación de la fuerza humana.

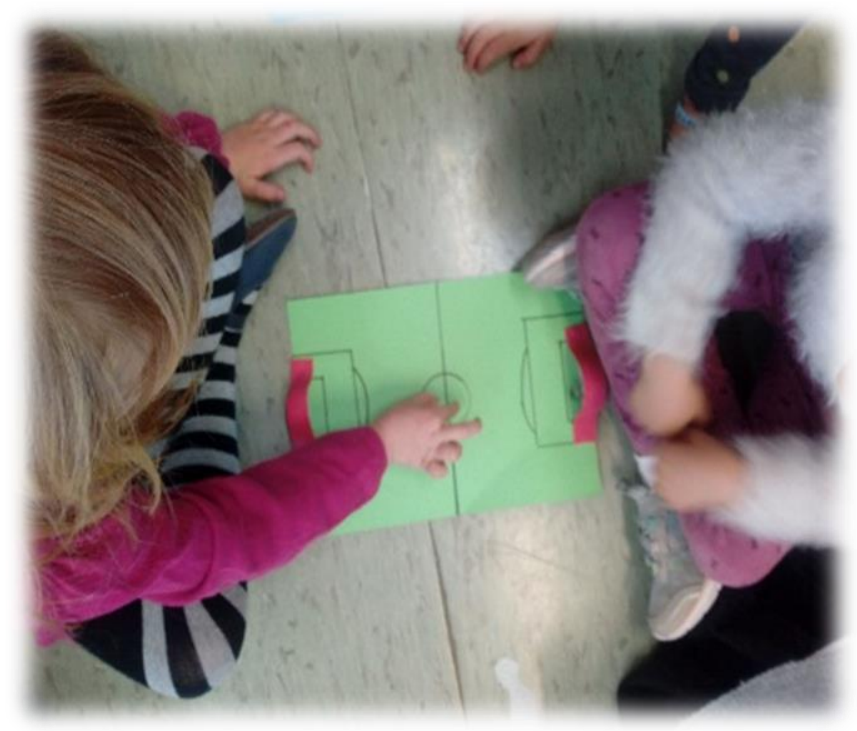

Ilustración 97. Investigación de la fuerza del aire.

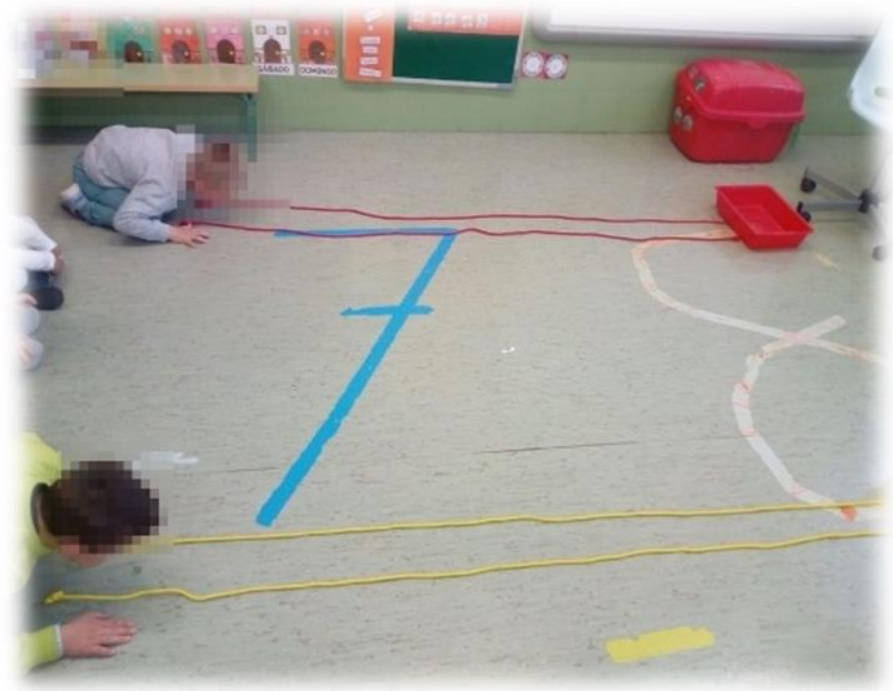


Ilustración 28. Investigación de la fuerza del aire

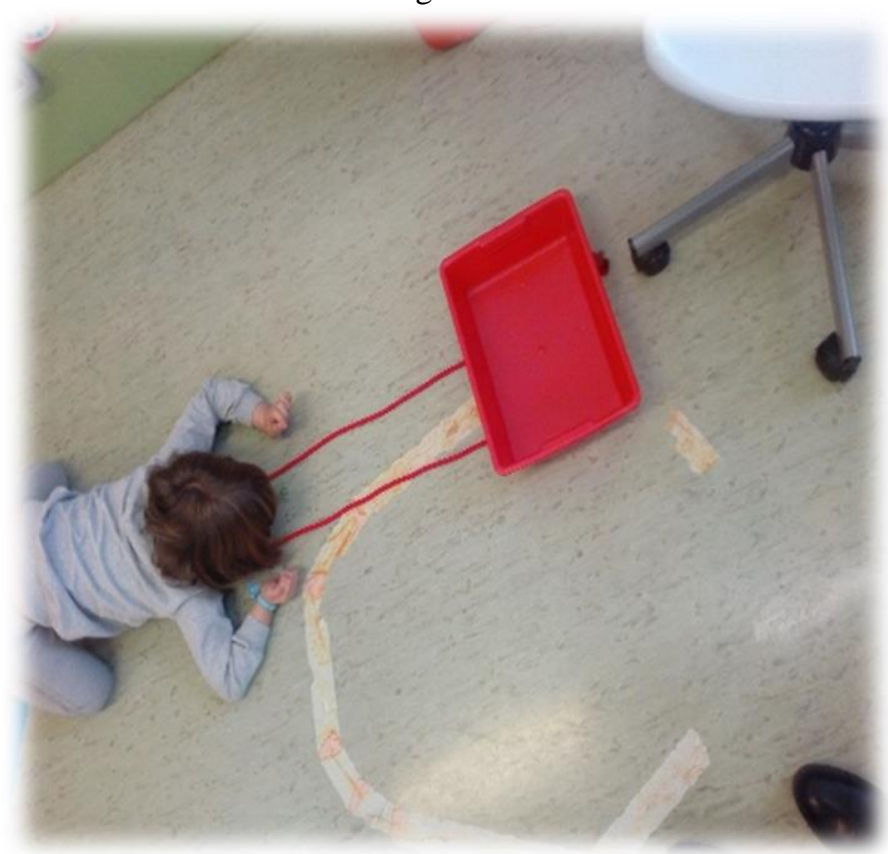

Ilustración 29. Investigación de la fuerza del agua.

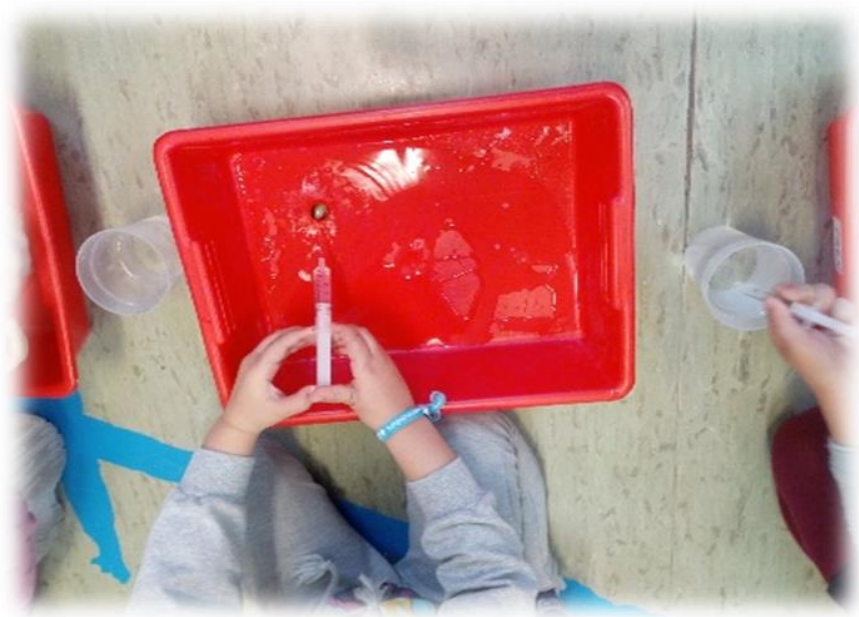

Ilustración 30. Investigación de la fuerza del agua.

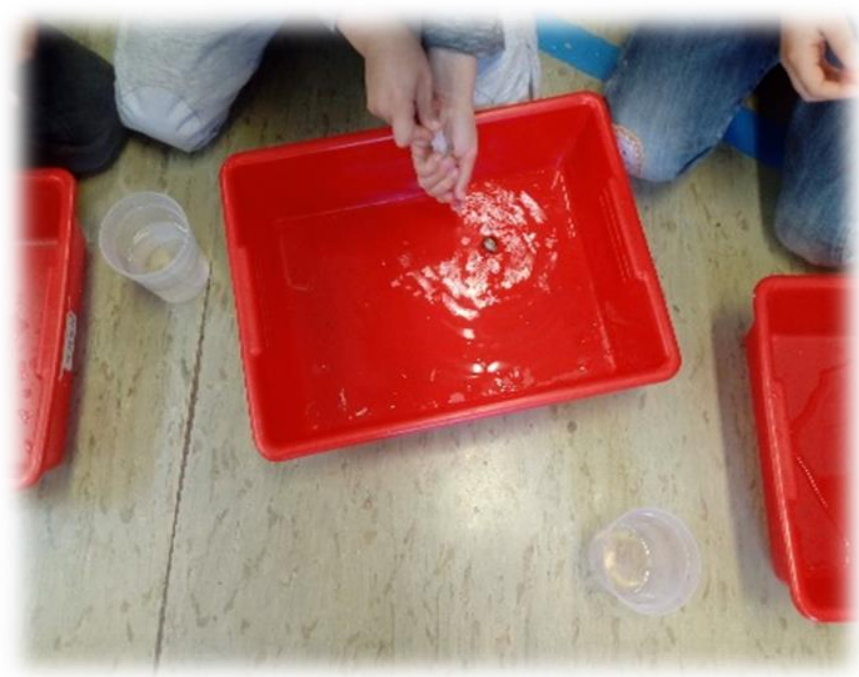


ANEXO XII

Ilustración 31. Recogida de datos de la investigación de la fuerza humana, la fuerza del aire y la fuerza del agua.

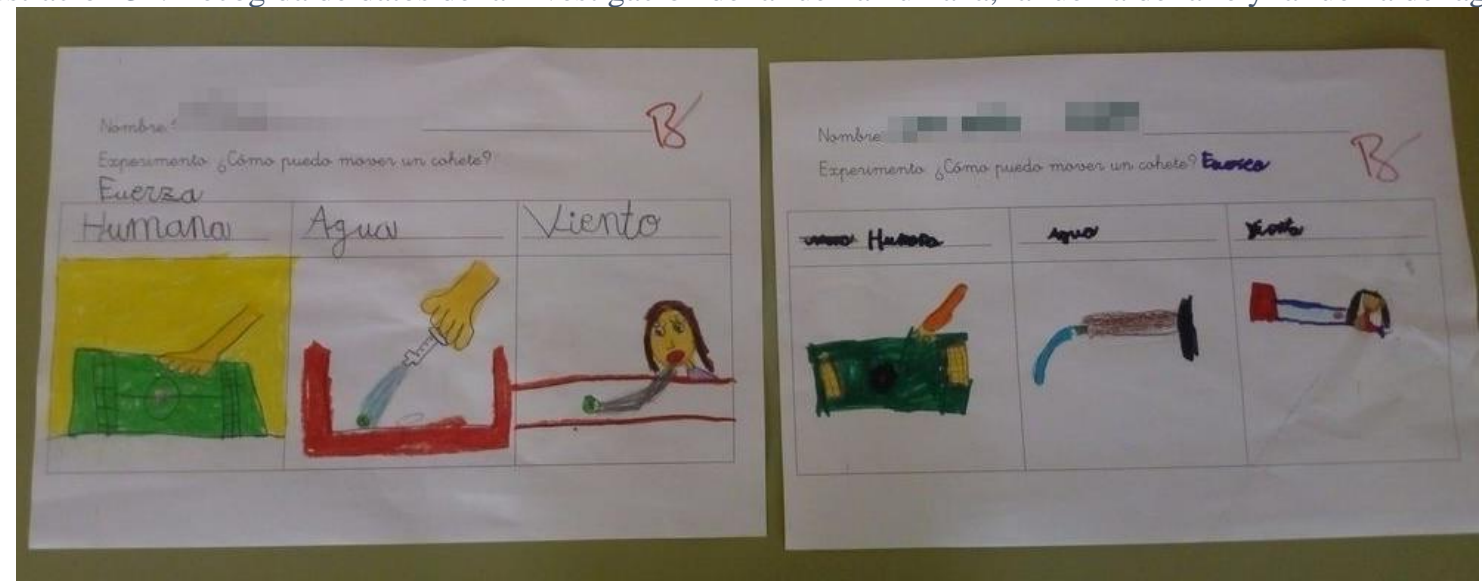

ANEXO XIII

Ilustración 32. Flashcards de la película "Wall-e: el batallón e limpieza"

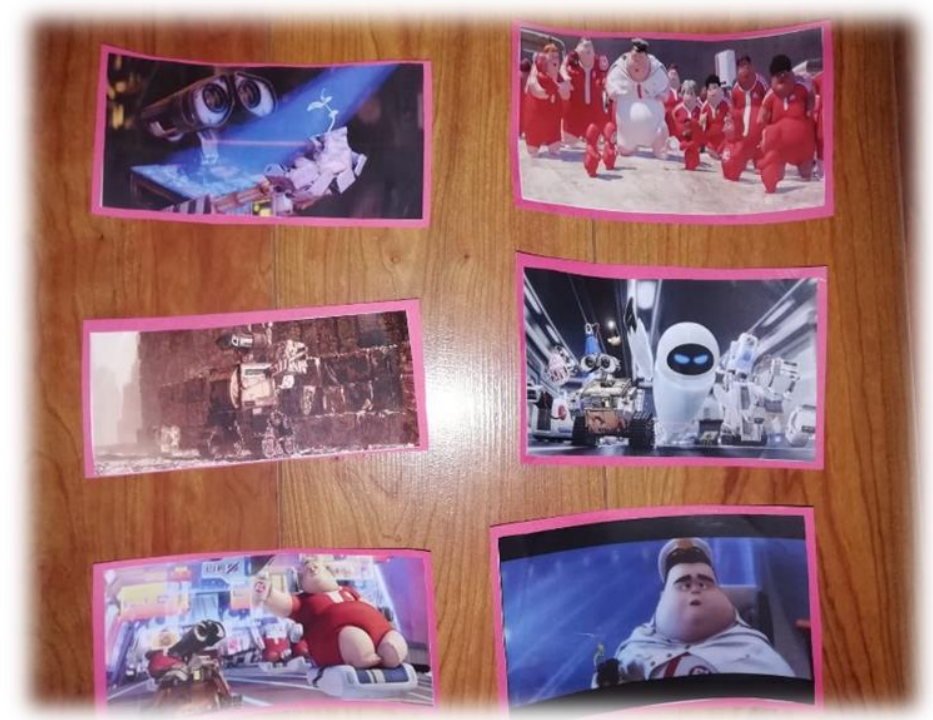

Ilustración 33. Dibujo de un transporte espacial recoge basura.

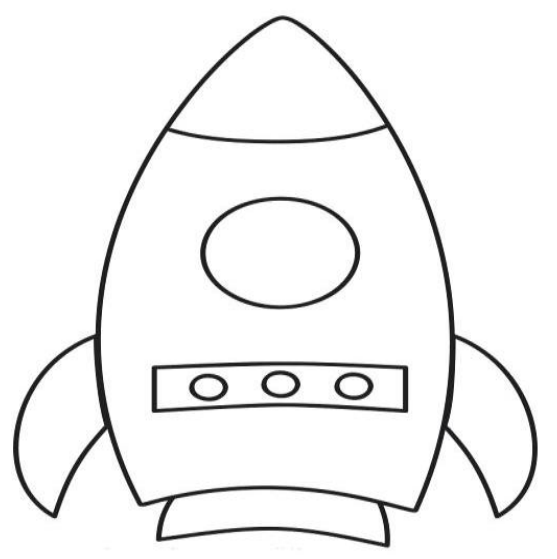




\section{ANEXO XIV}

Ilustración 34. Juego de "La guerra de limpieza"

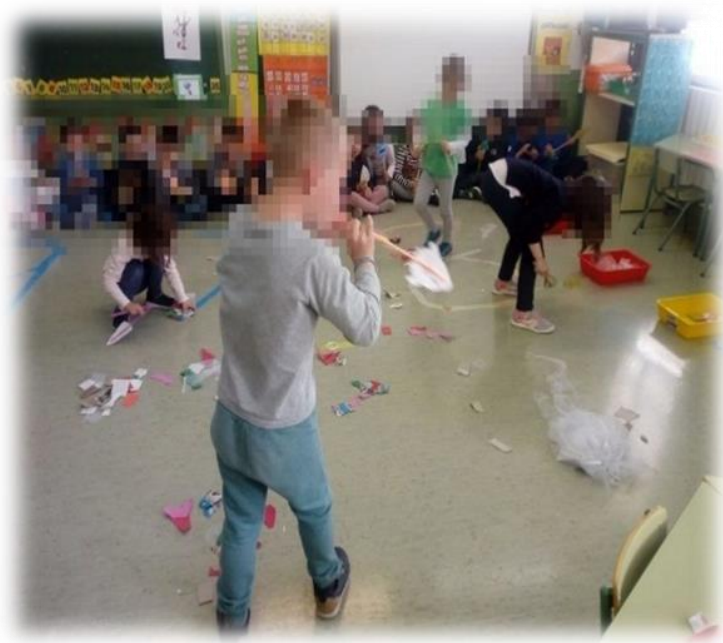

Ilustración 3510. Juego de "La guerra de limpieza"

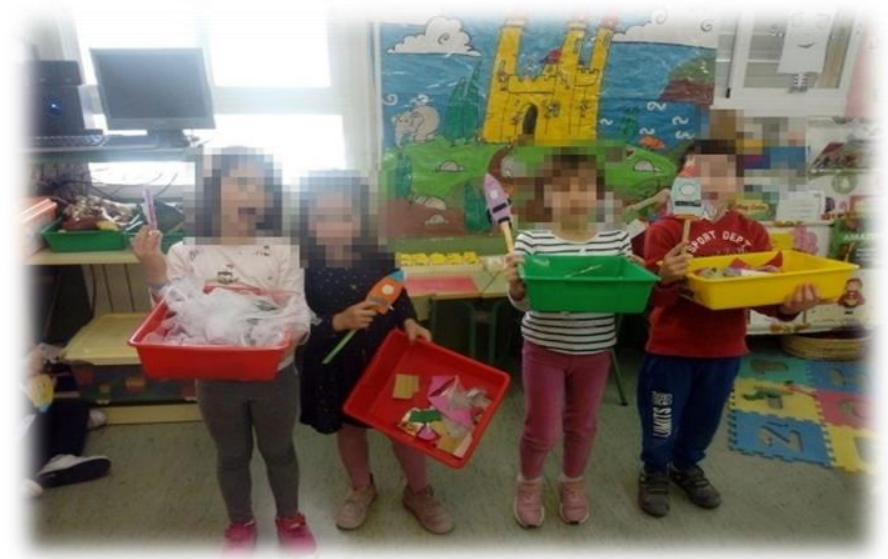

\section{ANEXO VX}

Ilustración 116. Mural donde se recoge los conocimientos que se van aprendiendo en la secuencia.

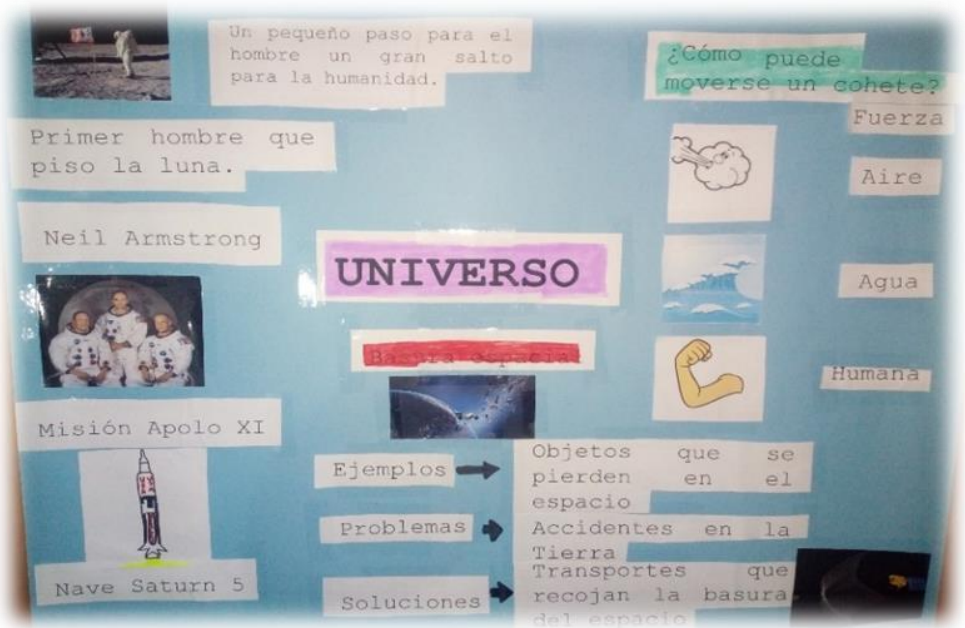


Ilustración 37. Mural donde se reflexiona y se obtienen las conclusiones de lo aprendido en la secuencia.

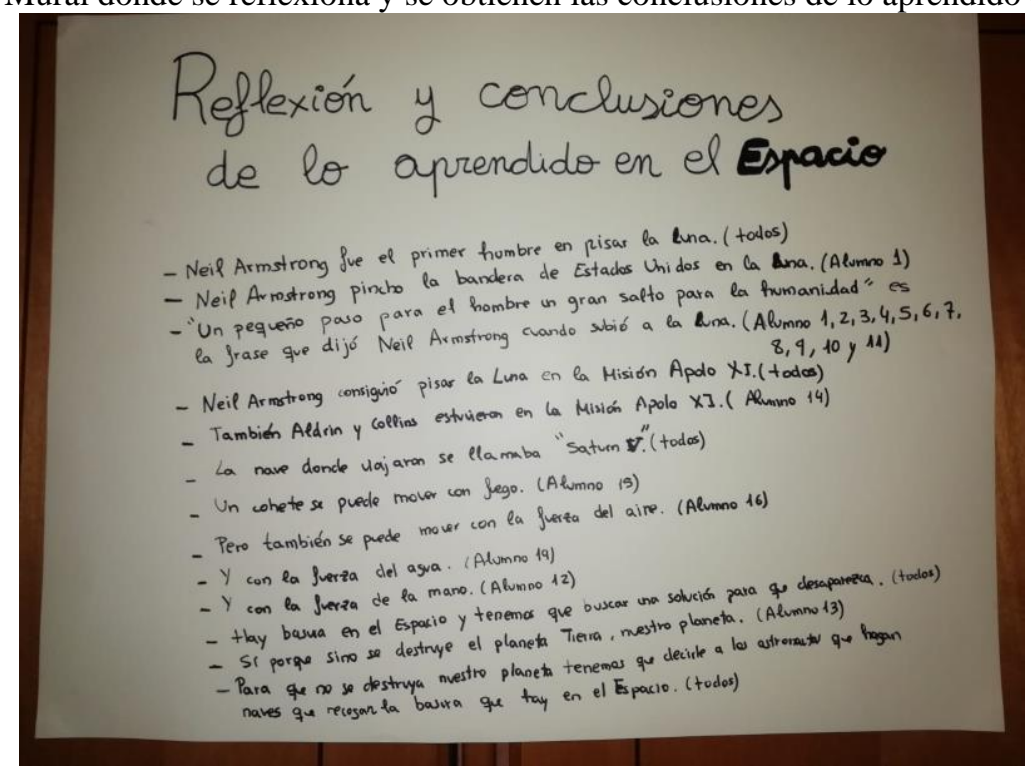

\section{ANEXO XVI}

https://create.kahoot.it/details/universo/5a872090-d861-41b4-9504-999570ae4ba5 